\title{
Energy levels, radiative rates, and electron impact excitation rates for transitions in $\mathrm{O} \mathrm{VII}^{\star}$
}

\author{
K. M. Aggarwal and F. P. Keenan
}

\author{
Astrophysics Research Centre, School of Mathematics and Physics, Queen's University Belfast, Belfast BT7 1NN, \\ Northern Ireland, UK \\ e-mail: K.Aggarwal@qub.ac.uk
}

Received 7 July 2008 / Accepted 7 August 2008

\begin{abstract}
Aims. In this paper we report calculations for energy levels, radiative rates, and electron impact excitation rates for transitions in O VII. Methods. The GRASP (general-purpose relativistic atomic structure package) is adopted for calculating energy levels and radiative rates. For determining the collision strengths and subsequently the excitation rates, the Dirac atomic R-matrix code (DARC) and the flexible atomic code (FAC) are used.

Results. Oscillator strengths, radiative rates, and line strengths are reported for all E1, E2, M1, and M2 transitions among the lowest 49 levels of O VII. Collision strengths have been averaged over a Maxwellian velocity distribution, and the resulting effective collision strengths are reported over a wide temperature range below $2 \times 10^{6} \mathrm{~K}$. Additionally, lifetimes are also listed for all levels.
\end{abstract}

Key words. atomic data - atomic processes

\section{Introduction}

Emission lines of He-like O VII have been widely observed in a variety of astrophysical and laboratory plasmas. For example, lines in the X-ray region (1-50 $\AA$ ) have been detected in solar flares by McKenzie et al. (1980) and Phillips et al. (1999), as listed by Dere et al. (2001). Similarly, Winkler et al. (1981) have observed some lines in the X-ray region from supernova remnants. Of particular interest are the resonance $\left(w: 1 s^{2}{ }^{1} \mathrm{~S}_{0}-1 \mathrm{~s} 2 \mathrm{p}\right.$ ${ }^{1} \mathrm{P}_{1}^{\circ}$ ), intercombination ( $x$ and $y: 1 \mathrm{~s}^{2}{ }^{1} \mathrm{~S}_{0}-1 \mathrm{~s} 2 \mathrm{p}^{3} \mathrm{P}_{2,1}^{\circ}$ ), and forbidden $\left(z: 1 \mathrm{~s}^{2}{ }^{1} \mathrm{~S}_{0}-1 \mathrm{~s} 2 \mathrm{~s}^{3} \mathrm{~S}_{1}\right)$ lines, which are highly useful for solar plasma diagnostics - see, for example, Gabriel \& Jordan (1969) and Acton et al. (1972). Keenan et al. (1985) have also shown that the $1 \mathrm{~s}^{2}{ }^{1} \mathrm{~S}-2 \mathrm{~s} n \mathrm{p}{ }^{1} \mathrm{P}(n=2-4)$ emission lines of $\mathrm{O}$ VII provide electron temperature estimates for the solar corona. Similarly, emission lines of O VII have been detected in the 15-140 A region by Isler et al. (1993), using spectra recorded at the ISX-A tokamak at Oak Ridge National Laboratory (ORNL), and by Baker (1993) in a theta-pinch plasma in the 1600-2500 ̊ wavelength range. However, to reliably analyse observations, atomic data are required for many parameters, such as: energy levels, radiative rates ( $A$-values), and excitation rates or equivalently the effective collision strengths $(\Upsilon)$, which are obtained from the electron impact collision strengths $(\Omega)$. Since experimental values are not available for the desired atomic parameters, except for energy levels, theoretical results are required.

Due to the wide variety of $\mathrm{O}$ VII observations and the diagnostic potential of lines from this ion, several calculations of atomic data are available in the literature. However, the most recent and comprehensive are by Delahaye \& Pradhan (2002),

* Tables 2 and 6 are only available in electronic form at the CDS via anonymous ftp to cdsarc.u-strasbg. fr $(130.79 .128 .5)$ or via http://cdsweb.u-strasbg.fr/cgi-bin/qcat? J/A+A/489/1377 who adopted the SuperStructure (SS) program of Eissner et al. (1974) for the generation of wavefunctions, and the Breit-Pauli $R$-matrix program of Berrington et al. (1995) for the computation of collision strengths $(\Omega)$ and subsequently effective collision strengths ( $\Upsilon$ ). They calculated values of $\Omega$ over a wide energy range below $200 \mathrm{Ryd}$, resolved resonances in the threshold region, and determined values of $\Upsilon$ over a wide temperature range of $10^{4}-10^{7} \mathrm{~K}$. Furthermore, they included one-body relativistic operators in the generation of wavefunctions as well as in the scattering process. Therefore, their results should be the most accurate available today. However, their calculations are confined to the $n \leq 4$ levels alone, whereas transitions involving the $n=5$ levels have been observed (Dere et al. 2001). Furthermore, Delahaye \& Pradhan presented only representative results for a few transitions, although their results for $\Upsilon$ are available electronically on the TIPbase (http://vizier.u-strasbg.fr/ tipbase/home.html). Therefore, our aim is to extend the calculations of Delahaye \& Pradhan by also including the $n=5$ levels, and to report a complete set of results (namely energy levels, radiative rates, and effective collision strengths) for all transitions among the lowest 49 levels of O VII. Finally, we also report the $A$-values for four types of transitions, namely electric dipole (E1), electric quadrupole (E2), magnetic dipole (M1), and magnetic quadrupole (M2), because these are also required for plasma modelling.

Furthermore, our approach is fully relativistic, as for the determination of wavefunctions we have employed the generalpurpose relativistic atomic structure package (GRASP), originally developed by Grant et al. (1980) and revised by Dr. Norrington. It is a fully relativistic code, based on the $j j$ coupling scheme. Further relativistic corrections arising from the Breit interaction and QED effects have also been included. Additionally, we have used the option of extended average level (EAL), in which a weighted (proportional to $2 j+1$ ) trace of the Hamiltonian matrix is minimized. This produces a compromise 
Table 1. Energy levels (in Ryd) of O VII.

\begin{tabular}{|c|c|c|c|c|c|c|c|c|c|}
\hline Index & 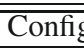 & iration/Level & NIST & GRASP1 & "GRASP2 & FAC1 & $\overline{\mathrm{FAC2}}$ & $\overline{\mathrm{SS}}$ & MBPT \\
\hline 1 & $1 s^{2}$ & ${ }^{1} \mathrm{~S}_{0}$ & 0.00000 & 0.00000 & 0.00000 & 0.00000 & 0.00000 & 0.0000 & 0.00000 \\
\hline 2 & $1 \mathrm{~s} 2 \mathrm{~s}$ & ${ }^{3} \mathrm{~S}_{1}$ & 41.23155 & 41.05246 & 41.03508 & 41.14868 & 41.14842 & 41.2438 & 41.23949 \\
\hline 3 & $1 s 2 p$ & ${ }^{3} \mathrm{P}_{0}^{\circ}$ & 41.78724 & 41.61135 & 41.59725 & 41.73910 & 41.73896 & 41.7933 & 41.79607 \\
\hline 4 & $1 s 2 p$ & ${ }^{3} \mathrm{P}_{1}^{\circ}$ & 41.78779 & 41.61434 & 41.59775 & 41.73960 & 41.73946 & 41.7942 & 41.79660 \\
\hline 5 & $1 \mathrm{~s} 2 \mathrm{p}$ & ${ }^{3} \mathrm{P}_{2}^{\circ}$ & 41.79280 & 41.62044 & 41.60273 & 41.74439 & 41.74425 & 41.7997 & 41.80161 \\
\hline 6 & $1 \mathrm{~s} 2 \mathrm{~s}$ & ${ }^{1} \mathrm{~S}_{0}^{2}$ & 41.81240 & 41.66296 & 41.64791 & 41.77554 & 41.77529 & 41.8074 & 41.81546 \\
\hline 7 & $1 \mathrm{~s} 2 \mathrm{p}$ & ${ }^{1} \mathrm{P}_{1}^{\circ}$ & 42.18438 & 42.01968 & 42.00142 & 42.17212 & 42.17198 & 42.2100 & 42.18790 \\
\hline 8 & $1 \mathrm{~s} 3 \mathrm{~s}$ & ${ }^{3} \mathrm{~S}_{1}$ & 48.65091 & 48.46935 & 48.45154 & 48.58117 & 48.58100 & 48.6577 & 48.66068 \\
\hline 9 & $1 \mathrm{~s} 3 \mathrm{p}$ & ${ }^{3} \mathrm{P}_{0}^{\circ}$ & 48.80446 & 48.62106 & 48.60415 & 48.73673 & 48.73664 & 48.8114 & 48.81207 \\
\hline 10 & $1 \mathrm{~s} 3 \mathrm{p}$ & ${ }^{3} \mathrm{P}_{1}^{\circ}$ & 48.80446 & 48.62193 & 48.60435 & 48.73697 & 48.73688 & 48.8116 & 48.81227 \\
\hline 11 & $1 \mathrm{~s} 3 \mathrm{p}$ & ${ }^{3} \mathrm{P}_{2}^{\circ}$ & 48.80446 & 48.62371 & 48.60583 & 48.73844 & 48.73835 & 48.8132 & 48.81376 \\
\hline 12 & $1 \mathrm{~s} 3 \mathrm{~s}$ & ${ }^{1} \mathrm{~S}_{0}^{2}$ & 48.81129 & 48.63498 & 48.61790 & 48.74376 & 48.74357 & 48.8217 & 48.81306 \\
\hline 13 & $1 \mathrm{~s} 3 \mathrm{~d}$ & ${ }^{3} \mathrm{D}_{1}$ & 48.88374 & 48.70037 & 48.68262 & 48.80681 & 48.80681 & 48.8930 & 48.89282 \\
\hline 14 & $1 \mathrm{~s} 3 \mathrm{~d}$ & ${ }^{3} \mathrm{D}_{2}$ & 48.88428 & 48.70065 & 48.68270 & 48.80689 & 48.80689 & 48.8931 & 48.89289 \\
\hline 15 & $1 \mathrm{~s} 3 \mathrm{~d}$ & ${ }^{3} \mathrm{D}_{3}$ & 48.88437 & 48.70116 & 48.68315 & 48.80732 & 48.80732 & 48.8935 & 48.89335 \\
\hline 16 & $1 \mathrm{~s} 3 \mathrm{~d}$ & ${ }^{1} \mathrm{D}_{2}$ & 48.89376 & 48.70506 & 48.68714 & 48.81229 & 48.81229 & 48.8971 & 48.89682 \\
\hline 17 & $1 \mathrm{~s} 3 \mathrm{p}$ & ${ }^{1} \mathrm{P}_{1}^{\circ}$ & 48.92183 & 48.73674 & 48.71873 & 48.85493 & 48.85482 & 48.9281 & 48.92119 \\
\hline 18 & $1 \mathrm{~s} 4 \mathrm{~s}$ & ${ }^{3} \mathrm{~S}_{1}$ & 51.17986 & 50.98944 & 50.97156 & 51.09360 & 51.09344 & 51.1813 & 51.18171 \\
\hline 19 & $1 \mathrm{~s} 4 \mathrm{p}$ & ${ }^{3} \mathrm{P}_{0}^{\circ}$ & 51.23690 & 51.05146 & 51.03394 & 51.14996 & 51.14988 & 51.2436 & 51.24367 \\
\hline 20 & $1 \mathrm{~s} 4 \mathrm{p}$ & ${ }^{3} \mathrm{P}_{1}^{\circ}$ & 51.23690 & 51.05183 & 51.03404 & 51.15008 & 51.15000 & 51.2437 & 51.24377 \\
\hline 21 & $1 \mathrm{~s} 4 \mathrm{p}$ & ${ }^{3} \mathrm{P}_{2}^{\circ}$ & 51.23690 & 51.05258 & 51.03466 & 51.15072 & 51.15063 & 51.2444 & 51.24440 \\
\hline 22 & $1 \mathrm{~s} 4 \mathrm{~s}$ & ${ }^{1} \mathrm{~S}_{0}^{2}$ & 51.24146 & 51.05811 & 51.04055 & 51.15922 & 51.15902 & 51.2475 & 51.24341 \\
\hline 23 & $1 \mathrm{~s} 4 \mathrm{~d}$ & ${ }^{3} \mathrm{D}_{1}$ & 51.26752 & 51.08411 & 51.06626 & 51.19366 & 51.19366 & 51.2767 & 51.27697 \\
\hline 24 & $1 \mathrm{~s} 4 \mathrm{~d}$ & ${ }^{3} \mathrm{D}_{2}$ & 51.26788 & 51.08424 & 51.06630 & 51.19370 & 51.19370 & 51.2767 & 51.27700 \\
\hline 25 & $1 \mathrm{~s} 4 \mathrm{~d}$ & ${ }^{3} \mathrm{D}_{3}$ & 51.27244 & 51.08445 & 51.06648 & 51.19385 & 51.19385 & 51.2769 & 51.27719 \\
\hline 26 & $1 \mathrm{~s} 4 \mathrm{f}$ & ${ }^{3} \mathrm{~F}_{2}^{\circ}$ & & 51.08597 & 51.06807 & 51.18727 & 51.18727 & 51.2786 & 51.27905 \\
\hline 27 & $1 \mathrm{~s} 4 \mathrm{f}$ & ${ }^{3} \mathrm{~F}_{3}^{\circ}$ & & 51.08598 & 51.06802 & 51.18723 & 51.18723 & 51.2785 & 51.27900 \\
\hline 28 & $1 \mathrm{~s} 4 \mathrm{f}$ & ${ }^{3} \mathrm{~F}_{4}^{\circ}$ & 51.26980 & 51.08614 & 51.06818 & 51.18739 & 51.18739 & 51.2787 & \\
\hline 29 & $1 \mathrm{~s} 4 \mathrm{f}$ & ${ }^{1} \mathrm{~F}_{3}^{\circ}$ & 51.27554 & 51.08615 & 51.06824 & 51.18746 & 51.18746 & 51.2787 & 51.27922 \\
\hline 30 & $1 \mathrm{~s} 4 \mathrm{~d}$ & ${ }^{1} \mathrm{D}_{2}^{3}$ & 51.27399 & 51.08662 & 51.06869 & 51.19656 & 51.19656 & 51.2790 & 51.27909 \\
\hline 31 & $1 \mathrm{~s} 4 \mathrm{p}$ & ${ }^{1} \mathrm{P}_{1}^{\circ}$ & 51.28702 & 51.09990 & 51.08194 & 51.19849 & 51.19838 & 51.2916 & 51.28866 \\
\hline 32 & $1 \mathrm{~s} 5 \mathrm{~s}$ & ${ }^{3} \mathrm{~S}_{1}$ & 52.33051 & 52.13981 & 52.12190 & 52.24041 & 52.24020 & & 52.33229 \\
\hline 33 & $1 \mathrm{~s} 5 \mathrm{p}$ & ${ }^{3} \mathrm{P}_{0}^{\circ}$ & 52.35630 & 52.17106 & 52.15333 & 52.26805 & 52.26793 & & 52.36350 \\
\hline 34 & $1 \mathrm{~s} 5 \mathrm{p}$ & ${ }^{3} \mathrm{P}_{1}^{\circ}$ & 52.35630 & 52.17125 & 52.15339 & 52.26811 & 52.26799 & & 52.36359 \\
\hline 35 & $1 \mathrm{~s} 5 \mathrm{p}$ & ${ }^{3} \mathrm{P}_{2}^{\circ}$ & 52.35630 & 52.17163 & 52.15371 & 52.26844 & 52.26831 & & 52.36398 \\
\hline 36 & $1 \mathrm{~s} 5 \mathrm{~s}$ & ${ }^{1} \mathrm{~S}_{0}^{2}$ & & 52.17550 & 52.15778 & 52.27342 & 52.27311 & & 52.36325 \\
\hline 37 & $1 \mathrm{~s} 5 \mathrm{~d}$ & ${ }^{3} \mathrm{D}_{1}$ & 52.37416 & 52.18755 & 52.16965 & 52.29583 & 52.29583 & & 52.38054 \\
\hline 38 & $1 \mathrm{~s} 5 \mathrm{~d}$ & ${ }^{3} \mathrm{D}_{2}$ & 52.37435 & 52.18761 & 52.16968 & 52.29585 & 52.29585 & & 52.38056 \\
\hline 39 & $1 \mathrm{~s} 5 \mathrm{~d}$ & ${ }^{3} \mathrm{D}_{3}$ & 52.37617 & 52.18772 & 52.16977 & 52.29593 & 52.29593 & & 52.38065 \\
\hline 40 & $1 \mathrm{~s} 5 \mathrm{f}$ & ${ }^{3} \mathrm{~F}_{2}^{\circ}$ & & 52.18858 & 52.17066 & 52.28959 & 52.28959 & & 52.38168 \\
\hline 41 & $1 \mathrm{~s} 5 \mathrm{f}$ & ${ }^{3} \mathrm{~F}_{3}^{\circ}$ & & 52.18858 & 52.17064 & 52.28958 & 52.28958 & & 52.38166 \\
\hline 42 & $1 \mathrm{~s} 5 \mathrm{f}$ & ${ }^{3} \mathrm{~F}_{4}^{\circ}$ & 52.37234 & 52.18866 & 52.17072 & 52.28965 & 52.28965 & & \\
\hline 43 & $1 \mathrm{~s} 5 \mathrm{f}$ & ${ }^{1} \mathrm{~F}_{3}^{\circ}$ & 52.37799 & 52.18867 & 52.17075 & 52.28969 & 52.28969 & & 52.38177 \\
\hline 44 & $1 \mathrm{~s} 5 \mathrm{~g}$ & ${ }^{3} \mathrm{G}_{3}$ & & 52.18867 & 52.17075 & 52.28956 & 52.28956 & & 52.38182 \\
\hline 45 & $1 \mathrm{~s} 5 \mathrm{~g}$ & ${ }^{3} \mathrm{G}_{4}$ & & 52.18867 & 52.17073 & 52.28955 & 52.28955 & & \\
\hline 46 & $1 \mathrm{~s} 5 \mathrm{~g}$ & ${ }^{3} \mathrm{G}_{5}$ & & 52.18872 & 52.17078 & 52.28960 & 52.28960 & & \\
\hline 47 & $1 \mathrm{~s} 5 \mathrm{~g}$ & ${ }^{1} \mathrm{G}_{4}$ & & 52.18872 & 52.17080 & 52.28962 & 52.28962 & & \\
\hline 48 & $1 \mathrm{~s} 5 \mathrm{~d}$ & ${ }^{1} \mathrm{D}_{2}$ & 52.38173 & 52.18895 & 52.17102 & 52.29745 & 52.29745 & & 52.38173 \\
\hline 49 & $1 \mathrm{~s} 5 \mathrm{p}$ & ${ }^{1} \mathrm{P}_{1}^{\circ}$ & 52.38373 & 52.19614 & 52.17819 & 52.29262 & 52.29246 & & 52.38634 \\
\hline
\end{tabular}

NIST: http://physics.nist.gov/PhysRefData. GRASP1: energies from the GRASP code with 49 level calculations without Breit and QED effects. GRASP2: energies from the GRASP code with 49 level calculations with Breit and QED effects. FAC1: energies from the FAC code with 49 level calculations. FAC2: energies from the FAC code with 71 level calculations. SS: energies of Delahaye \& Pradhan (2002) from the sS code. MBPT: energies of Savukov et al. (2003) from the MBPT code.

set of orbitals describing closely lying states with moderate accuracy. Similarly, for our calculations of $\Omega$, we have adopted the Dirac atomic R-matrix code (DARC) of Norrington \& Grant (private communication). Finally, in order to assess the accuracy of our results, we have performed parallel calculations from the Flexible Atomic Code (FAC) of Gu (2003), available from the website http://kipac-tree.stanford.edu/fac. This is also a fully relativistic code which provides a variety of atomic parameters, and yields results comparable to GRASP and DARC.
Thus, results from FAC will be helpful in assessing the accuracy of our energy levels, radiative rates, and collision strengths.

\section{Energy levels}

The $1 \mathrm{~s}^{2}, 1 \mathrm{~s} 2 \ell, 1 \mathrm{~s} 3 \ell, 1 \mathrm{~s} 4 \ell$, and $1 \mathrm{~s} 5 \ell$ configurations of $\mathrm{O}$ VII give rise to the lowest 49 levels listed in Table 1, where we compare our level energies from GRASP, obtained without and with the inclusion of Breit and QED effects, with the experimental 
values compiled by NIST (National Institute of Standards and Technology), and available at their website http://physics . nist.gov/PhysRefData. Our level energies obtained without Breit and QED effects (GRASP1) are consistently lower than the experimental values by $\sim 0.2 \mathrm{Ryd}$, but are in agreement within $0.5 \%$. However, the orderings are slightly different from those of NIST in a few instances, such as for levels 16, 30, and 42. The inclusion of Breit and QED effects (slightly) lowers the level energies by $\sim 0.02$ Ryd (GRASP2), and hence comparatively increases the difference with the experimental values, but the agreement remains within $0.5 \%$. Furthermore, the orderings have slightly altered in three instances, namely for levels $26 / 27$, 40/41, and 43/45. However, the energy differences for these "swapping" levels are very small. Our level energies obtained from the FAC code (FAC1), including the same CI (configuration interaction) as in GRASP1, are consistently higher by $\sim 0.1$ Ryd and hence are comparatively in better agreement with the NIST listings. The level orderings from FAC1 are also in agreement with our calculations from GRASP, except for the $1 \mathrm{~s} 5 \mathrm{~d}{ }^{1} \mathrm{D}_{2}$ and $1 \mathrm{~s} 5 \mathrm{p}{ }^{1} \mathrm{P}_{1}^{\circ}$ levels (48 and 49). For these, our orderings from GRASP are in agreement with the experimental values. Other minor differences in level orderings from FAC1 are for the levels of the $1 \mathrm{~s} 5 \mathrm{f}$ and $1 \mathrm{~s} 5 \mathrm{~g}$ configurations. A further inclusion of $1 \mathrm{~s} 6 \ell$ configurations, as in the FAC 2 calculations, makes no difference either in magnitude or orderings, mainly because levels of the $1 \mathrm{~s} 6 \ell$ configurations lie above the lowest 49 levels listed in Table 1, and hence do not interact with these.

Other energy levels listed in Table 1 are from the SS calculations of Delahaye \& Pradhan (2002) and those of Savukov et al. (2003) from relativistic many-body perturbation theory (MBPT). The energy levels of Delahaye \& Pradhan are in better agreement with the experimental values, but are available only for the lowest 31 levels. Additionally, if we have a closer look at the energies of $1 \mathrm{~s} 2 \mathrm{p}^{3} \mathrm{P}_{0,1,2}^{\circ}$, the level splittings of Delahaye \& Pradhan differ considerably with the experimental and other theoretical results. Similarly, the energy levels of Savukov et al. are available for all the $J \leq 3$ levels, and are in better agreement with the experimental results, but only in magnitude. Their level orderings are different with our calculations and the NIST listings in many instances, such as for levels: 12, 22, 30, and particularly for level $48\left(1 \mathrm{~s} 5 \mathrm{~d}^{1} \mathrm{D}_{2}\right)$. Since most of the energy levels within any $n$ complex are very close to one another, different calculations provide slightly different orderings. To conclude, we may state that overall there is no discrepancy between theory and experiment for the energy levels of O VII. However, experimental energies are not available for all the levels listed in Table 1, and the $1 \mathrm{~s} 3 \mathrm{p}^{3} \mathrm{P}_{0,1,2}^{\circ}, 1 \mathrm{~s} 4 \mathrm{p}{ }^{3} \mathrm{P}_{0,1,2}^{\circ}$, and $1 \mathrm{~s} 5 \mathrm{p}{ }^{3} \mathrm{P}_{0,1,2}^{\circ}$ levels are non-degenerate in energy. For such non-degenerate and missing levels, we recommend that our energy levels either from the GRASP2 or FAC1 calculations should be adopted in any plasma modelling applications. For the remaining levels, the experimentally compiled listings of the NIST should be preferred.

\section{Radiative rates}

The absorption oscillator strength $\left(f_{i j}\right)$ and radiative rate $A_{j i}$ (in $\mathrm{s}^{-1}$ ) for a transition $i \rightarrow j$ are related by the following expression:

$f_{i j}=\frac{m c}{8 \pi^{2} e^{2}} \lambda_{j i}^{2} \frac{\omega_{j}}{\omega_{i}} A_{j i}=1.49 \times 10^{-16} \lambda_{j i}^{2}\left(\omega_{j} / \omega_{i}\right) A_{j i}$

where $m$ and $e$ are the electron mass and charge, respectively, $c$ is the velocity of light, $\lambda_{j i}$ is the transition energy/wavelength in $\AA$, and $\omega_{i}$ and $\omega_{j}$ are the statistical weights of the lower $(i)$ and upper $(j)$ levels, respectively. Similarly, the oscillator strength $f_{i j}$ (dimensionless) and the line strength $S$ (in atomic unit) are related by the standard equations listed below.

For the electric dipole (E1) transitions

$A_{j i}=\frac{2.0261 \times 10^{18}}{\omega_{j} \lambda_{j i}^{3}} S^{\mathrm{E} 1} \quad$ and $\quad f_{i j}=\frac{303.75}{\lambda_{j i} \omega_{i}} S^{\mathrm{E} 1}$

for the magnetic dipole (M1) transitions

$A_{j i}=\frac{2.6974 \times 10^{13}}{\omega_{j} \lambda_{j i}^{3}} S^{\mathrm{M} 1} \quad$ and $\quad f_{i j}=\frac{4.044 \times 10^{-3}}{\lambda_{j i} \omega_{i}} S^{\mathrm{M} 1}$,

for the electric quadrupole (E2) transitions

$A_{j i}=\frac{1.1199 \times 10^{18}}{\omega_{j} \lambda_{j i}^{5}} S^{\mathrm{E} 2} \quad$ and $\quad f_{i j}=\frac{167.89}{\lambda_{j i}^{3} \omega_{i}} S^{\mathrm{E} 2}$

and for the magnetic quadrupole (M2) transitions

$A_{j i}=\frac{1.4910 \times 10^{13}}{\omega_{j} \lambda_{j i}^{5}} S^{\mathrm{M} 2} \quad$ and $\quad f_{i j}=\frac{2.236 \times 10^{-3}}{\lambda_{j i}^{3} \omega_{i}} S^{\mathrm{M} 2}$

In Table 2 we present transition energies/wavelengths ( $\lambda$, in $\AA$ ), radiative rates $\left(A_{j i}\right.$, in s $\left.{ }^{-1}\right)$, oscillator strengths $\left(f_{i j}\right.$, dimensionless), and line strengths ( $S$, in au), in length form only, for all 336 electric dipole (E1) transitions among the 49 levels of O VII. The indices used to represent the lower and upper levels of a transition have already been defined in Table 1. Similarly, there are 391 electric quadrupole (E2), 316 magnetic dipole (M1), and 410 magnetic quadrupole (M2) transitions among the 49 levels. However, for these transitions only the $A$-values are listed in Table 2, and the corresponding results for $f$ - or $S$-values can be easily obtained using Eqs. (1)-(5).

In Table 3 we compare our radiative rates ( $A$-values), both from GRASP and FAC, with those of Delahaye \& Pradhan (2002) from the ss code, Savukov et al. (2003) from MBPT, and of those listed on the NIST website, for the common E1 transitions. Generally, all sets of $A$-values agree within $10 \%$, although for some weak transitions, such as: $2-5,6-7$, and $12-17$, differences are up to $20 \%$, and for the $16-17\left(1 \mathrm{~s} 3 \mathrm{~d}^{1} \mathrm{D}_{2}-1 \mathrm{~s} 3 \mathrm{p}{ }^{1} \mathrm{P}_{1}^{\circ}\right.$, $f=0.0085)$ transition, the discrepancy is up to a factor of two. For this transition our $A$-value is the highest whereas those of Savukov et al. is the lowest. This is because weak transitions are very sensitive to mixing coefficients, and hence differing amount of CI (and methods) produce different $A$-values, as discussed in detail by Hibbert (2000). However, we would like to emphasize here that although A-values for weak transitions are also required in modelling applications, their contribution is usually not very important in comparison to stronger transitions with $f \geq 0.01$.

One of the general criteria to assess the accuracy of radiative rates is to compare the length and velocity forms of the $f$ - or $A$-values. However, such comparisons are only desirable, and are not a fully sufficient test to assess accuracy, as different calculations (or combinations of configurations) may give comparable $f$-values in the two forms, but entirely different results in magnitude. Generally, there is a good agreement between the length and velocity forms of the $f$-values for strong transitions, but differences between the two forms can sometimes be substantial even for some very strong transitions, as demonstrated through 
Table 3. Comparison of $A$-values (in $\mathrm{s}^{-1}$ ) for some transitions of O VII. $a \pm b \equiv a \times 10^{ \pm b}$.

\begin{tabular}{rrrrrrr}
\hline \hline$i$ & $j$ & NIST & GRASP & FAC & SS & MBPT \\
\hline 1 & 7 & $3.309+12$ & $3.4949+12$ & $3.408+12$ & $3.403+12$ & $3.302+12$ \\
1 & 17 & $9.365+11$ & $1.1027+12$ & $9.958+11$ & $1.004+12$ & $9.345+11$ \\
2 & 3 & $7.797+07$ & $8.3043+07$ & $9.537+07$ & $8.058+07$ & $7.940+07$ \\
2 & 4 & $7.820+07$ & $8.3276+07$ & $9.562+07$ & $8.083+07$ & $7.964+07$ \\
2 & 5 & $8.033+07$ & $8.5585+07$ & $9.807+07$ & $8.309+07$ & $8.188+07$ \\
3 & 8 & $2.505+09$ & $2.4036+09$ & $2.311+09$ & $2.237+09$ & $2.519+09$ \\
3 & 13 & $8.982+10$ & $8.9564+10$ & $8.814+10$ & $8.927+10$ & $8.969+10$ \\
4 & 8 & $7.512+09$ & $7.1885+09$ & $6.912+09$ & $6.739+09$ & $7.538+09$ \\
4 & 13 & $6.735+10$ & $6.7164+10$ & $6.610+10$ & $6.699+10$ & $6.726+10$ \\
4 & 14 & $1.213+11$ & $1.1973+11$ & $1.183+11$ & $1.205+11$ & $1.196+11$ \\
5 & 8 & $1.249+10$ & $1.2021+10$ & $1.156+10$ & $1.131+10$ & $1.261+10$ \\
5 & 13 & $4.481+09$ & $4.4734+09$ & $4.402+09$ & $4.466+09$ & $4.480+09$ \\
5 & 14 & $4.033+10$ & $3.9761+10$ & $3.930+10$ & $4.012+10$ & $3.969+10$ \\
5 & 15 & $1.613+11$ & $1.6109+11$ & $1.586+11$ & $1.608+11$ & $1.613+11$ \\
6 & 7 & $2.514+07$ & $2.1567+07$ & & $2.509+07$ & $2.548+07$ \\
6 & 17 & $5.055+10$ & $5.2792+10$ & $5.121+10$ & $5.209+10$ & $5.039+10$ \\
7 & 12 & $2.008+10$ & $2.2082+10$ & $2.077+10$ & $2.223+10$ & $2.014+10$ \\
7 & 16 & $1.523+11$ & $1.5139+11$ & $1.487+11$ & $1.540+11$ & $1.503+11$ \\
9 & 13 & $6.114+05$ & $5.9952+05$ & & $6.200+05$ & $6.523+05$ \\
10 & 13 & $4.585+05$ & $4.4635+05$ & & $4.649+05$ & $4.857+05$ \\
10 & 14 & $8.426+05$ & $7.9793+05$ & & $8.535+05$ & $8.656+05$ \\
11 & 13 & $3.057+04$ & $2.8024+04$ & & $3.099+04$ & $3.054+04$ \\
11 & 14 & $2.809+05$ & $2.5043+05$ & & $2.841+05$ & $2.719+05$ \\
11 & 15 & $1.127+06$ & $1.0327+06$ & & $1.143+06$ & $1.125+06$ \\
12 & 17 & $3.864+06$ & $3.0023+06$ & & $3.958+06$ & $3.739+06$ \\
16 & 17 & $7.410+04$ & $1.1423+05$ & & $8.082+04$ & $5.238+08$ \\
\hline & & & & &
\end{tabular}

NIST: http://physics.nist.gov/PhysRefData. GRASP: present 49 level calculations from the GRASP code. FAC: present 49 level calculations from the FAC code. SS: calculations of Delahaye \& Pradhan (2002) from the ss code. MBPT: calculations of Savukov et al. (2003) from the MBPT code.

various examples by Aggarwal et al. (2007). Nevertheless, for almost all of the strong transitions $(f \geq 0.01)$ the two forms agree to within $20 \%$, but differences for $11(<4 \%)$ transitions are higher by up to a factor of two. Additionally, for two transitions (6-7: $f=0.064$ and 48-49: $f=0.023$ ), the two forms differ by factors of 3 and 6 , respectively. However, for both of these transition energies $(\Delta E)$ are very small, and a slight variation in $\Delta E$ affects the $A$-values considerably. Therefore, on the basis of these comparisons and discussion we may state that for a majority of the strong E1 transitions, our radiative rates are accurate to better than $20 \%$. However, for the weaker transitions this assessment of accuracy does not apply.

\section{Lifetimes}

The lifetime $\tau$ for a level $j$ is defined as follows:

$\tau_{j}=\frac{1}{\sum_{i} A_{j i}}$.

Since this is a measurable parameter, it provides a check on the accuracy of the calculations. Therefore, in Table 4 we have listed our calculated lifetimes, which include the contributions from four types of transitions, i.e. E1, E2, M1, and M2. Also included in this table are the theoretical results of Savukov et al. (2003) from MBPT and some early measurements by Träbert et al. (1977) from beam-foil spectra.

In general, agreement between our present and earlier (Savukov et al. 2003) theoretical lifetimes is better than $20 \%$ for most of the levels, but the differences are larger for a few levels, such as 6,36 , and 49 . Since for level $6\left(1 \mathrm{~s} 2 \mathrm{~s}{ }^{1} \mathrm{~S}_{0}\right)$ both the magnitude of the lifetime as well as the discrepancy is the highest (over an order of magnitude), we focus on this level alone.
The maximum contribution for this level comes from the 4-6 E1 transition, for which our $A$-value is $25.69 \mathrm{~s}^{-1}$, whereas that of Savukov et al. is $1.435 \mathrm{~s}^{-1}$. However, the $4-6$ transition is very weak $\left(f=4.24 \times 10^{-7}\right)$, and hence accuracy estimates are always insecure (Hibbert 2000). Nevertheless, our above $f$-value from GRASP compares reasonably well (within a factor of two) with the corresponding FAC calculations, but the corresponding difference in the $A$-values is a factor of three. This is because the $A$ - and $f$-values have a $\Delta E^{2}$ (or equivalently $\lambda_{j i}^{2}$ ) dependence as seen already in Eq. (1), and therefore any difference in $\Delta E$ has a larger effect on the $A$-value. The experimental value of $\Delta E$ for the 4-6 transition is $0.02461 \mathrm{Ryd}$, and the corresponding values from GRASP, FAC, and MBPT are 0.0502, 0.03594, and 0.01886 Ryd, respectively. Therefore, an approximate "correction" to the $A$-values can be applied (Hibbert 1996) by multiplying these by the $\left(\Delta E_{\mathrm{exp}} / \Delta E_{\mathrm{the}}\right)^{2}$ factor. Following this, the $A$-values from GRASP, FAC, and MBPT are $6.174 \mathrm{~s}^{-1}, 3.671 \mathrm{~s}^{-1}$, and $2.443 \mathrm{~s}^{-1}$, or the $\tau$ values are $0.162 \mathrm{~s}, 0.272 \mathrm{~s}$, and $0.409 \mathrm{~s}$, respectively. As a result, the discrepancy for the $\tau$ values among three independent calculations is now within a factor of 2.5. A measurement of a lifetime for the $1 \mathrm{~s} 2 \mathrm{~s}{ }^{1} \mathrm{~S}_{0}$ level will be helpful to resolve the discrepancy.

For the levels for which experimental values of $\tau$ are available, the agreement is within the limits of uncertainty, except for level $8\left(1 \mathrm{~s} 3 \mathrm{~s}^{3} \mathrm{~S}_{1}\right)$ for which the discrepancy is $\sim 40 \%$. Overall we may state that there is good agreement between the theoretical and experimental lifetimes for a majority of the levels.

\section{Collision strengths}

For the computation of collision strengths $\Omega$, we have employed the Dirac atomic R-matrix code (DARC), which includes the 
Table 4. Comparison of theoretical and experimental lifetimes ( $\tau$ in s) for the levels of O VII. $a-b \equiv a \times 10^{-b}$.

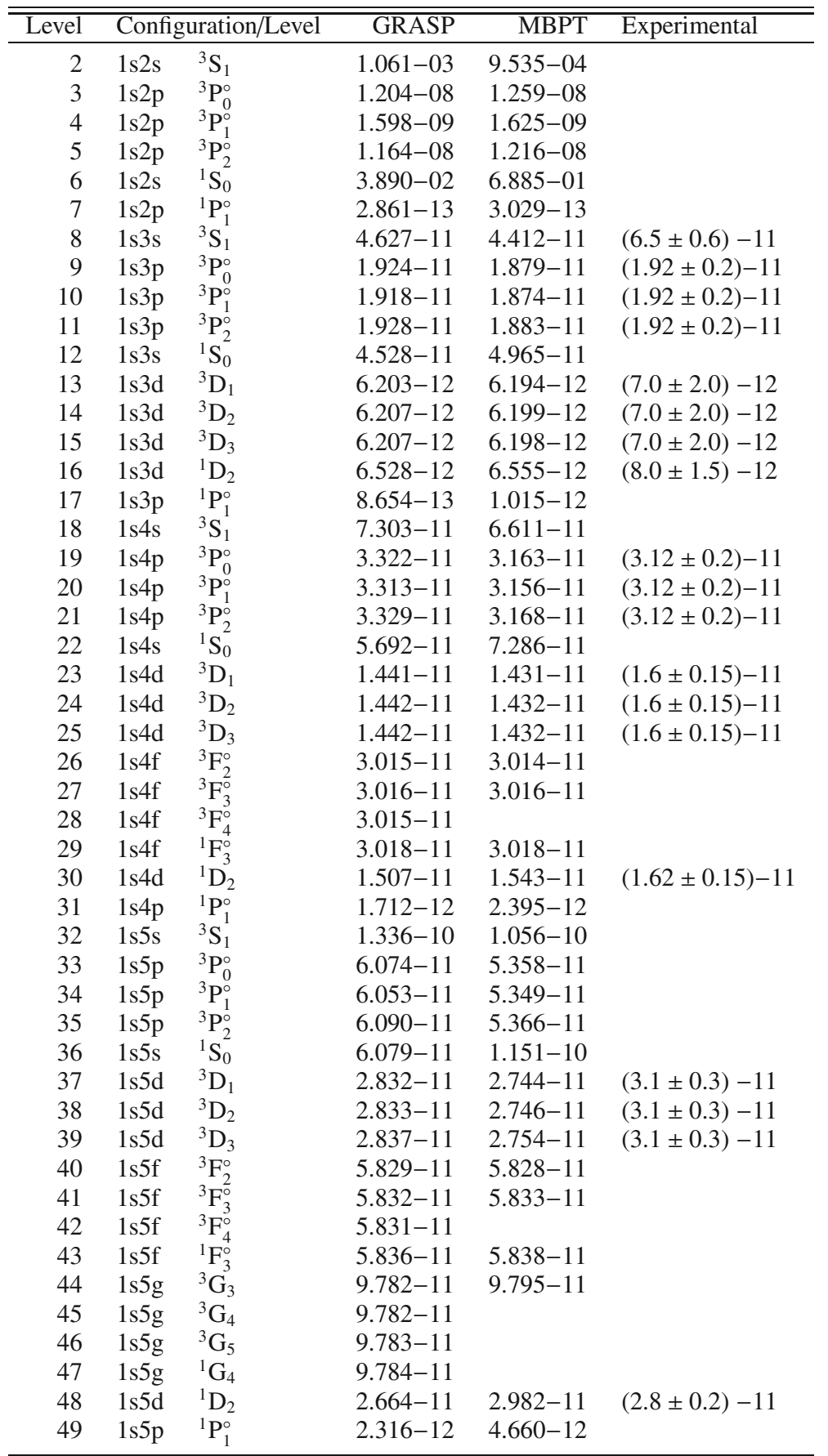

GRASP: Present 49 level calculations from the GRASP code. MBPT: calculations of Savukov et al. (2003) from the MBPT code. Expt: measurements for the $L S$ states by Trabert et al. (1977).

relativistic effects in a systematic way, in both the target description and the scattering model. It is based on the $j j$ coupling scheme, and uses the Dirac-Coulomb Hamiltonian in the $R$-matrix approach. The $R$-matrix radius has been adopted to be $14.24 \mathrm{au}$, and 55 continuum orbitals have been included for each channel angular momentum for the expansion of the wavefunction. This allows us to compute $\Omega$ up to an energy of 80 Ryd, sufficient to calculate the excitation rates up to a temperature of $10^{6} \mathrm{~K}$. The maximum number of channels for a partial wave is 217 , and the corresponding size of the Hamiltonian matrix is 11991. In order to obtain convergence of $\Omega$ for all transitions and at all energies, we have included all partial waves with angular momentum $J \leq 40.5$, although a larger number would have been preferable for the convergence of some allowed transitions, especially at higher energies. However, to account for the inclusion of higher neglected partial waves, we have included a top-up, based on the Coulomb-Bethe approximation for allowed transitions and geometric series for others.

In Figs. $1-3$ we show the variation of $\Omega$ with angular momentum $J$ for three transitions, namely $2-4\left(1 \mathrm{~s} 2 \mathrm{~s}^{3} \mathrm{~S}_{1}-1 \mathrm{~s} 2 \mathrm{p}^{3} \mathrm{P}_{1}^{\circ}\right)$, 2-10 (1s $\left.2 \mathrm{~s}^{3} \mathrm{~S}_{1}-1 \mathrm{~s} 3 \mathrm{p}{ }^{3} \mathrm{P}_{1}^{\circ}\right)$, and 9-11 (1s3p $\left.{ }^{3} \mathrm{P}_{0}^{\circ}-1 \mathrm{~s} 3 \mathrm{p}^{3} \mathrm{P}_{2}^{\circ}\right)$, 


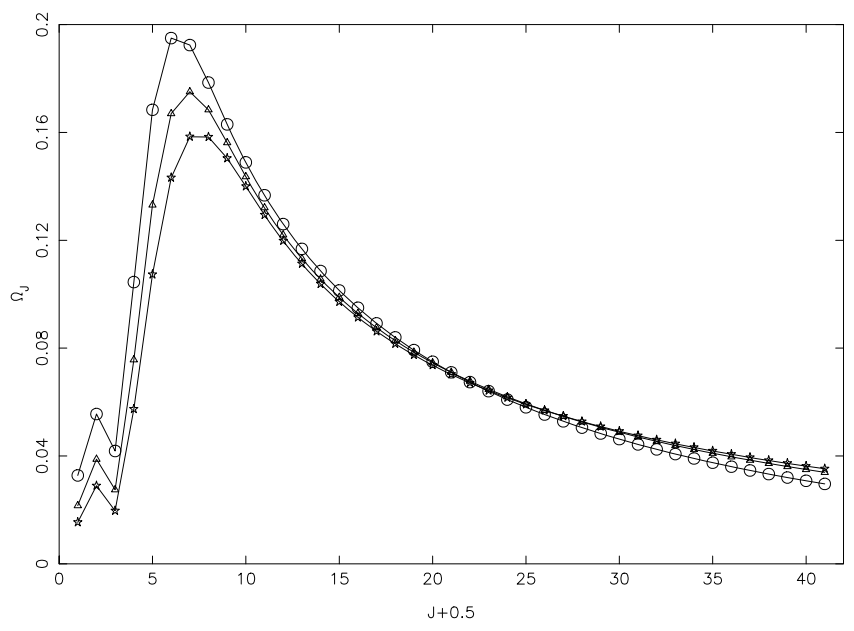

Fig. 1. Partial collision strengths for the $1 \mathrm{~s} 2 \mathrm{~s}^{3} \mathrm{~S}_{1}-1 \mathrm{~s} 2 \mathrm{p}{ }^{3} \mathrm{P}_{1}^{\circ}(2-4)$ transition of O VII, at three energies of: 60 Ryd (circles), 70 Ryd (triangles), and 80 Ryd (stars).

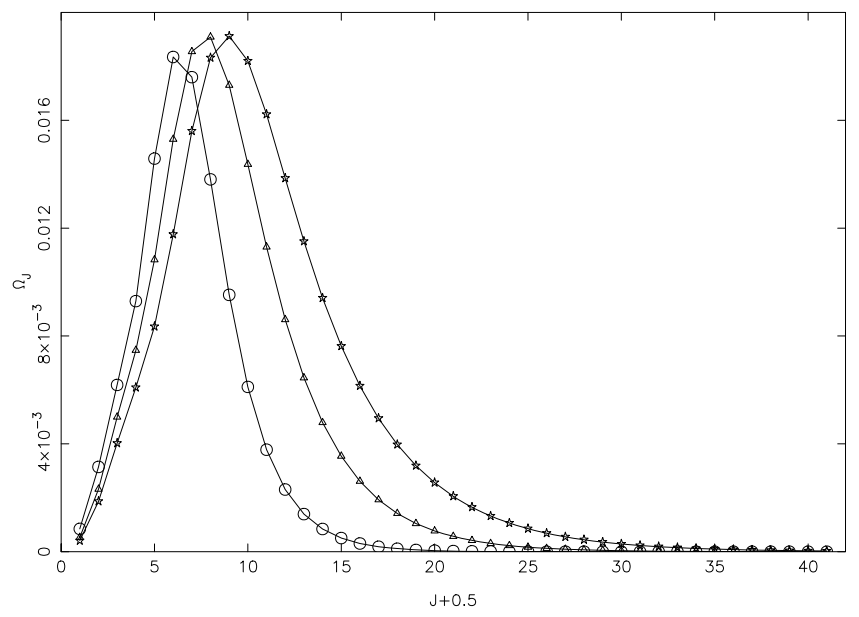

Fig. 2. Partial collision strengths for the $1 \mathrm{~s} 2 \mathrm{~s}{ }^{3} \mathrm{~S}_{1}-1 \mathrm{~s} 3 \mathrm{p}{ }^{3} \mathrm{P}_{1}^{\circ}(2-10)$ transition of O VII, at three energies of: 60 Ryd (circles), 70 Ryd (triangles), and 80 Ryd (stars).

respectively, and at three energies of 60,70 , and 80 Ryd. Values of $\Omega$ have fully converged for all resonance transitions, including the allowed ones. Values of $\Omega$ have also converged for allowed transitions among the higher excited levels, as shown in Fig. 2 for the 2-10 transition. It is also clear from Fig. 2 that the need to include a larger range of partial waves increases with increasing energy. However, values of $\Omega$ have not converged for those allowed transitions whose $\Delta E$ is very small (mainly within the same $n$ complex), as shown for the 2-4 transition in Fig. 1. Similarly, values of $\Omega$ have (almost) converged for all forbidden transitions, including those whose $\Delta E$ is very small, such as the 9-11 transition shown in Fig. 3. Therefore, only for the allowed transitions within the same $n$ complex, our wide range of partial waves is not sufficient for the convergence of $\Omega$, for which a top-up has been included as mentioned above.

In Table 5 we list our values of $\Omega$ for resonance transitions at energies above thresholds. The indices used to represent the levels of a transition have already been defined in Table 1. No comparisons can be made with our calculations because Delahaye $\&$ Pradhan (2002) have not reported results for collision strengths. Therefore, in order to make an accuracy assessment of the

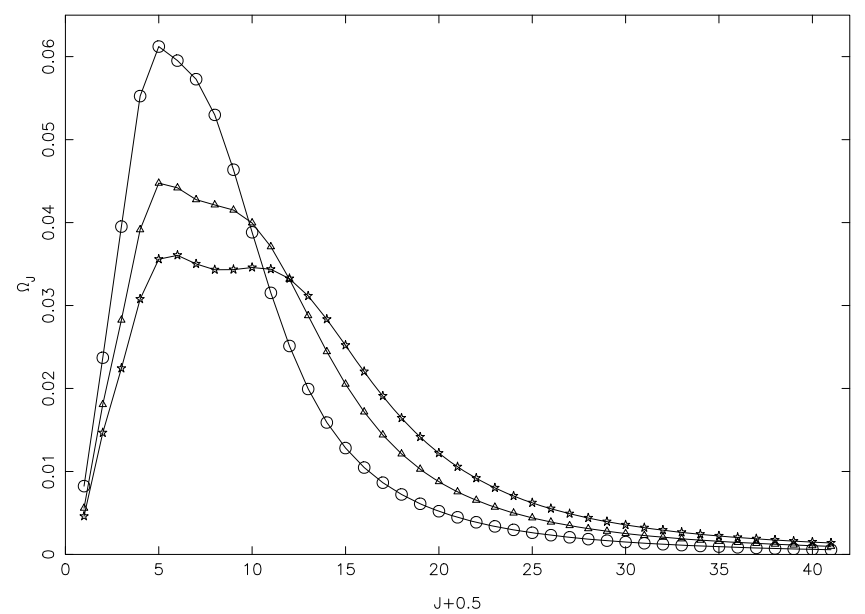

Fig. 3. Partial collision strengths for the $1 \mathrm{~s} 3 \mathrm{p}^{3} \mathrm{P}_{0}^{\circ}-1 \mathrm{~s} 3 \mathrm{p}^{3} \mathrm{P}_{2}^{\circ}(9-11)$ transition of O VII, at three energies of: 60 Ryd (circles), 70 Ryd (triangles), and 80 Ryd (stars).

values of $\Omega$, we have performed another calculation using the FAC code of $\mathrm{Gu}$ (2003). This code is also fully relativistic, and is based on the well known and widely used distorted-wave (DW) method. Furthermore, the same CI is included in FAC as in the calculations from DARC. Therefore, also included in Table 5 for a ready comparison are the $\Omega$ values from FAC at a single $e x$ cited energy $\left(E_{j}\right)$ of $\sim 75 \mathrm{Ryd}$, corresponding to the incident energy of $\sim 120$ Ryd. Generally the two sets of $\Omega$ agree well, but differences for four transitions, namely 1-27, 1-29, 1-41, and $1-43$, are over $30 \%$, and the discrepancy is an order of magnitude for two transitions, namely $1-45$ and $1-47$. However, all these transitions are very weak $\left(\Omega \leq 10^{-6}\right)$. The contribution of such weak transitions in any modelling application is likely to be insignificant.

In Fig. 4 we show the variation of our values of $\Omega$ with energy for three allowed transitions, namely $2-5\left(1 \mathrm{~s} 2 \mathrm{~s}{ }^{3} \mathrm{~S}_{1}-\right.$ $\left.1 \mathrm{~s} 2 \mathrm{p}^{3} \mathrm{P}_{2}^{\circ}\right), 4-14\left(1 \mathrm{~s} 2 \mathrm{p}^{3} \mathrm{P}_{1}^{\circ}-1 \mathrm{~s} 3 \mathrm{~d}^{3} \mathrm{D}_{2}\right)$, and $10-24\left(1 \mathrm{~s} 3 \mathrm{p}^{3} \mathrm{P}_{1}^{\circ}-1 \mathrm{~s} 4 \mathrm{~d}\right.$ $\left.{ }^{3} \mathrm{D}_{2}\right)$. Also included in this figure are the corresponding results obtained from the FAC code. It may be noted that our calculations from DARC are only up to $E=140$ Ryd (see Sect. 6) whereas the FAC calculates $\Omega$ up to $\sim 500$ Ryd. For all the above three (and many other) transitions there are no discrepancies between the $f$-values obtained from the two independent (GRASP and FAC) codes, and therefore the $\Omega$ values also agree to better than $20 \%$. However, the $\Omega$ values obtained from FAC are slightly anomalous, particularly towards the lower end of the energy range, and the agreement between the two calculations improves with increasing energy. Such occasional anomalies for a few random transitions occur because of the interpolation and extrapolation techniques employed in the FAC code, which is designed to generate a large amount of atomic data in a comparatively very short period of time, and without too much loss of accuracy. Similarly, some difference in the values of $\Omega$ are expected because the DW method generally overestimates the results for light ions due to the exclusion of channel coupling.

Similar comparisons between the two calculations are made in Fig. 5 for three forbidden transitions, namely $2-8\left(1 \mathrm{~s} 2 \mathrm{~s}^{3} \mathrm{~S}_{1-}\right.$ $\left.1 \mathrm{~s} 3 \mathrm{~s}{ }^{3} \mathrm{~S}_{1}\right), 2-15\left(1 \mathrm{~s} 2 \mathrm{~s}{ }^{3} \mathrm{~S}_{1}-1 \mathrm{~s} 3 \mathrm{~d}^{3} \mathrm{D}_{3}\right)$, and $4-10\left(1 \mathrm{~s} 2 \mathrm{p}{ }^{3} \mathrm{P}_{1}^{\circ}-1 \mathrm{~s} 3 \mathrm{p}\right.$ $\left.{ }^{3} \mathrm{P}_{1}^{\circ}\right)$. For these transitions, the agreement between the two calculations also improves with increasing energy. Therefore, in some instances a problem of a few anomalies may arise from the calculations from FAC, but overall we observe no discrepancy with our 
Table 5. Collision strengths for resonance transitions of O VII. $\left(a \pm b \equiv a \times 10^{ \pm b}\right)$.

\begin{tabular}{|c|c|c|c|c|c|c|c|c|}
\hline \multicolumn{2}{|c|}{ Transition } & \multicolumn{7}{|c|}{ Energy (Ryd) } \\
\hline$i$ & $j$ & 60 & 70 & 80 & 100 & 120 & 140 & FAC \\
\hline 1 & 2 & $2.786-3$ & $2.322-3$ & $1.942-3$ & $1.461-3$ & $1.129-3$ & $9.092-4$ & $1.089-3$ \\
\hline 1 & 3 & $1.534-3$ & $1.193-3$ & $9.460-4$ & $6.141-4$ & $4.273-4$ & $3.109-4$ & $3.973-4$ \\
\hline 1 & 4 & $4.598-3$ & $3.576-3$ & $2.837-3$ & $1.845-3$ & $1.287-3$ & $9.400-4$ & $1.201-3$ \\
\hline 1 & 5 & $7.628-3$ & $5.927-3$ & $4.699-3$ & $3.048-3$ & $2.120-3$ & $1.542-3$ & $1.980-3$ \\
\hline 1 & 6 & $9.337-3$ & $1.016-2$ & $1.077-2$ & $1.180-2$ & $1.252-2$ & $1.305-2$ & $1.178-2$ \\
\hline 1 & 7 & $3.701-2$ & $4.592-2$ & $5.412-2$ & $6.785-2$ & $7.978-2$ & $9.023-2$ & $8.016-2$ \\
\hline 1 & 8 & $9.065-4$ & $7.043-4$ & $5.721-4$ & $4.155-4$ & $3.167-4$ & $2.523-4$ & $2.709-4$ \\
\hline 1 & 9 & $4.878-4$ & $3.748-4$ & $2.948-4$ & $1.894-4$ & $1.306-4$ & $9.421-5$ & $1.063-4$ \\
\hline 1 & 10 & $1.462-3$ & $1.123-3$ & $8.837-4$ & $5.681-4$ & $3.925-4$ & $2.839-4$ & $3.203-4$ \\
\hline 1 & 11 & $2.427-3$ & $1.864-3$ & $1.466-3$ & $9.406-4$ & $6.484-4$ & $4.674-4$ & $5.298-4$ \\
\hline 1 & 12 & $1.821-3$ & $2.023-3$ & $2.180-3$ & $2.467-3$ & $2.682-3$ & $2.845-3$ & $2.493-3$ \\
\hline 1 & 13 & $8-4$ & $8-4$ & $7.754-5$ & $4.267-5$ & $2.659-5$ & -5 & $2.385-5$ \\
\hline 1 & 14 & $2.744-4$ & $1.859-4$ & $1.334-4$ & $7.735-5$ & $5.201-5$ & $3.866-5$ & $4.706-5$ \\
\hline 1 & 15 & 3.8 & $8-4$ & $1.798-4$ & $9.892-5$ & $6.161-5$ & -5 & $5.555-5$ \\
\hline 1 & 16 & 5.0 & $5.912-4$ & $6.933-4$ & $8.814-4$ & $1.033-3$ & -3 & $9.166-4$ \\
\hline 1 & 17 & 6.4 & $8.257-3$ & $9.894-3$ & 1.2 & $1.484-2$ & -2 & $1.597-2$ \\
\hline 1 & 18 & $4.280-4$ & $3.094-4$ & $2.452-4$ & $1.734-4$ & $1.294-4$ & -4 & $1.054-4$ \\
\hline 1 & 19 & $2.115-4$ & $1.613-4$ & $1.264-4$ & $8.045-5$ & $5.517-5$ & 3.9 & $4.254-5$ \\
\hline 1 & 20 & $6.338-4$ & $4.836-4$ & $3.788-4$ & $2.414-4$ & $1.657-4$ & $1.195-4$ & $1.283-4$ \\
\hline 1 & 21 & $1.053-3$ & $8.029-4$ & $6.284-4$ & $3.998-4$ & $2.740-4$ & $1.970-4$ & $2.119-4$ \\
\hline 1 & 22 & $7.265-4$ & $7.901-4$ & $8.585-4$ & $9.626-4$ & $1.046-3$ & $1.114-3$ & $9.677-4$ \\
\hline 1 & 23 & $9.500-5$ & $6.304-5$ & $4.438-5$ & $2.424-5$ & $1.504-5$ & $1.012-5$ & $1.299-5$ \\
\hline 1 & 24 & $1.586-4$ & $1.057-4$ & $7.498-5$ & $4.202-5$ & $2.718-5$ & $1.934-5$ & $2.354-5$ \\
\hline 1 & 25 & $2.206-4$ & $1.463-4$ & $1.029-4$ & $5.617-5$ & $3.486-5$ & -5 & $3.027-5$ \\
\hline 1 & 26 & $5.495-6$ & $3.063-6$ & $1.960-6$ & $5-7$ & $5.344-7$ & -7 & $19-7$ \\
\hline 1 & 27 & 1. & $1-6$ & $5-6$ & -6 & $5-6$ & & $3.308-6$ \\
\hline 1 & 28 & & -6 & -6 & -6 & $3-7$ & & $4-7$ \\
\hline 1 & 29 & & -6 & $5-6$ & -6 & -6 & & $5-6$ \\
\hline 1 & 30 & & -4 & -4 & 4.0 & & & $2-4$ \\
\hline 1 & 31 & -3 & $3.130-3$ & $3.749-3$ & $4.787-3$ & 5.6 & -3 & $6.029-3$ \\
\hline 1 & 32 & $7-4$ & $1.643-4$ & 1.2 & $8.751-5$ & 6.4 & 5.1 & $5.025-5$ \\
\hline 1 & 33 & $1.122-4$ & $8.365-5$ & $6.473-5$ & $4.097-5$ & $2.793-5$ & -5 & $2.073-5$ \\
\hline 1 & 34 & $3.362-4$ & $2.507-4$ & $1.941-4$ & $1.229-4$ & $8.392-5$ & $6.035-5$ & $6.253-5$ \\
\hline 1 & 35 & $5.586-4$ & $4.163-4$ & $3.220-4$ & $2.036-4$ & $1.387-4$ & $9.951-5$ & $1.032-4$ \\
\hline 1 & 36 & $4.065-4$ & $4.309-4$ & $4.595-4$ & $5.167-4$ & $5.554-4$ & $5.908-4$ & $4.970-4$ \\
\hline 1 & 37 & $5.569-5$ & $3.623-5$ & $2.536-5$ & $1.381-5$ & $8.550-6$ & $5.745-6$ & $7.286-6$ \\
\hline 1 & 38 & $9.290-5$ & $6.063-5$ & $4.269-5$ & $2.370-5$ & $1.515-5$ & $1.065-5$ & $1.292-5$ \\
\hline 1 & 39 & $1.293-4$ & $8.409-5$ & $5.883-5$ & $3.201-5$ & $1.981-5$ & $1.331-5$ & $1.697-5$ \\
\hline 1 & 40 & $4.671-6$ & $2.547-6$ & $1.615-6$ & $7.794-7$ & $4.374-7$ & $2.731-7$ & $3.762-7$ \\
\hline 1 & 41 & $9.145-6$ & $5.904-6$ & $4.703-6$ & $3.801-6$ & $3.500-6$ & $3.377-6$ & $2.311-6$ \\
\hline 1 & 42 & $0-6$ & $4.562-6$ & $2.891-6$ & $1.395-6$ & $7.826-7$ & $85-7$ & $6.763-7$ \\
\hline 1 & 43 & $1.221-5$ & $1-6$ & $7.571-6$ & $6.983-6$ & $6.891-6$ & $4-6$ & $4.364-6$ \\
\hline 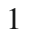 & 44 & $1.595-7$ & $5.615-8$ & $2.942-8$ & $1.143-8$ & $5.888-9$ & $3.599-9$ & $4.133-9$ \\
\hline 1 & 45 & & $1.829-7$ & $8-7$ & $1.416-7$ & 2.44 & 3.5 & $2.197-8$ \\
\hline 1 & 46 & $2.497-7$ & $8.783-8$ & $4.600-8$ & $1.786-8$ & $9.206-9$ & $5.626-9$ & $6.489-9$ \\
\hline 1 & 47 & $4.071-7$ & $2.113-7$ & $1.546-7$ & $1.740-7$ & $3.047-7$ & $4.392-7$ & $2.526-8$ \\
\hline 1 & 48 & & $1.528-4$ & $1.689-4$ & $2.100-4$ & $2.490-4$ & $2.842-4$ & $2.356-4$ \\
\hline 1 & 49 & $1.299-3$ & $1.643-3$ & $1.956-3$ & $2.477-3$ & $2.926-3$ & $3.306-3$ & $3.024-3$ \\
\hline
\end{tabular}

results from the DARC code, as also found for many other ions, such as those of iron - see, for example, Aggarwal et al. (2008) and references therein. In conclusion, based on the discussion above and the comparisons made, we do not see any apparent deficiency in our calculations for $\Omega$, and estimate our results to be accurate to better than $20 \%$ for a majority of the transitions.

\section{Excitation rates}

Excitation rates, along with energy levels and radiative rates, are required for plasma modelling, and are determined from the collision strengths $(\Omega)$. Since the threshold energy region is dominated by numerous closed-channel (Feshbach) resonances, values of $\Omega$ need to be calculated in a fine energy mesh in order to accurately account for their contribution. Furthermore, in a hot plasma electrons have a wide distribution of velocities, and therefore values of $\Omega$ are generally averaged over a Maxwellian distribution as follows:

$\Upsilon\left(T_{\mathrm{e}}\right)=\int_{0}^{\infty} \Omega(E) \exp \left(-E_{j} / k T_{\mathrm{e}}\right) \mathrm{d}\left(E_{j} / k T_{\mathrm{e}}\right)$

where $k$ is Boltzmann constant, $T_{\mathrm{e}}$ is the electron temperature in $\mathrm{K}$, and $E_{j}$ is the electron energy with respect to the final (excited) state. Once the value of $\Upsilon$ is known the corresponding results for 


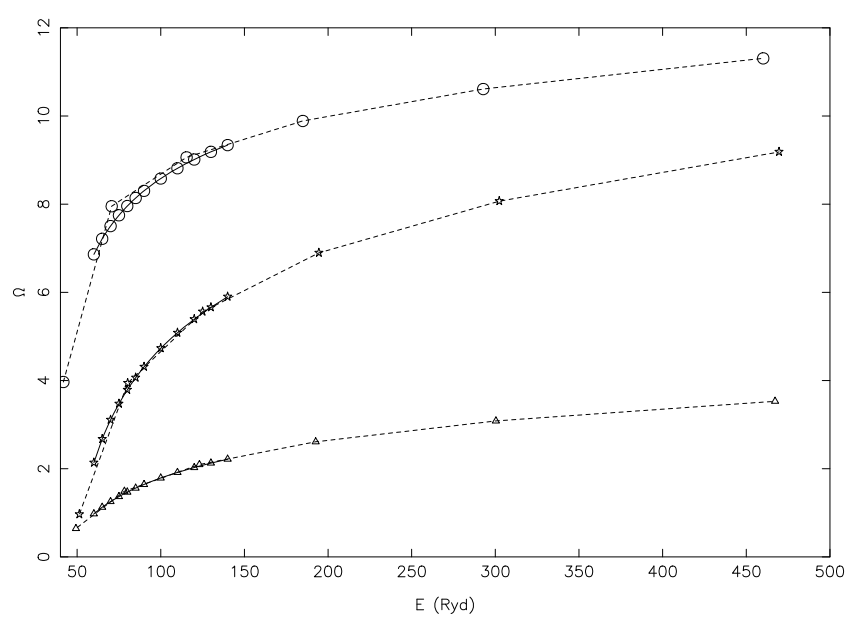

Fig. 4. Comparison of collision strengths from our calculations from DARC (continuous curves) and FAC (broken curves) for the 2-5 (circles: $1 \mathrm{~s} 2 \mathrm{~s}{ }^{3} \mathrm{~S}_{1}-1 \mathrm{~s} 2 \mathrm{p}{ }^{3} \mathrm{P}_{2}^{\circ}$ ), 4-14 (triangles: $1 \mathrm{~s} 2 \mathrm{p}{ }^{3} \mathrm{P}_{1}^{\circ}-1 \mathrm{~s} 3 \mathrm{~d}{ }^{3} \mathrm{D}_{2}$ ), and $10-24$ (stars: $1 \mathrm{~s} 3 \mathrm{p}^{3} \mathrm{P}_{1}^{\circ}-1 \mathrm{~s} 4 \mathrm{~d}^{3} \mathrm{D}_{2}$ ) allowed transitions of $\mathrm{O}$ VII .

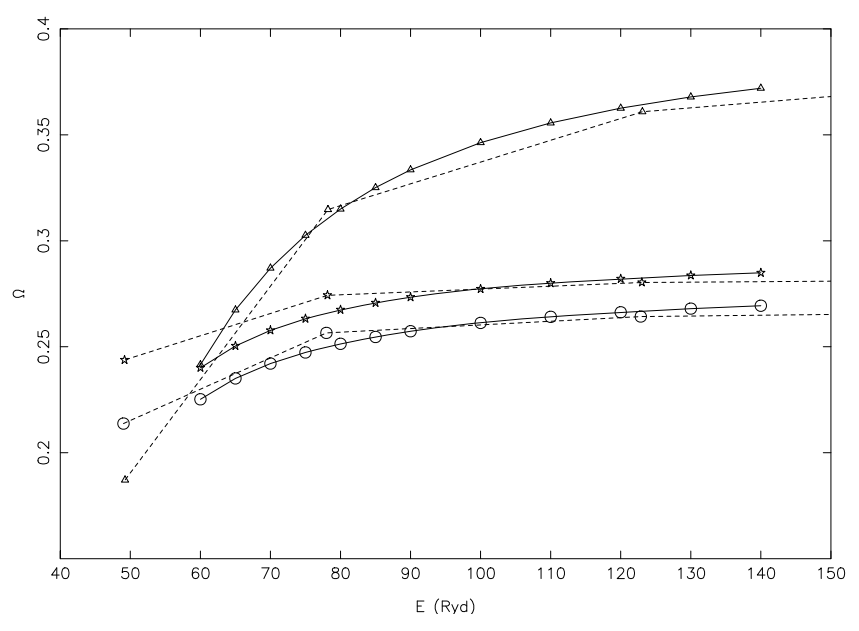

Fig. 5. Comparison of collision strengths from our calculations from DARC (continuous curves) and FAC (broken curves) for the 2-8 (circles: $1 \mathrm{~s} 2 \mathrm{~s}^{3} \mathrm{~S}_{1}-1 \mathrm{~s} 3 \mathrm{~s}^{3} \mathrm{~S}_{1}$ ), 2-15 (triangles: $1 \mathrm{~s} 2 \mathrm{~s}{ }^{3} \mathrm{~S}_{1}-1 \mathrm{~s} 3 \mathrm{~d}^{3} \mathrm{D}_{3}$ ), and $4-10$ (stars: $1 \mathrm{~s} 2 \mathrm{p}^{3} \mathrm{P}_{1}^{\circ}-1 \mathrm{~s} 3 \mathrm{p}^{3} \mathrm{P}_{1}^{\circ}$ ) forbidden transitions of $\mathrm{O}$ VII.

the excitation $q(i, j)$ and de-excitation $q(j, i)$ rates can be easily obtained from the following equations:

$q(i, j)=\frac{8.63 \times 10^{-6}}{\omega_{i} T_{\mathrm{e}}^{1 / 2}} \Upsilon \exp \left(-E_{i j} / k T_{\mathrm{e}}\right) \quad \mathrm{cm}^{3} \mathrm{~s}^{-1}$

and

$q(j, i)=\frac{8.63 \times 10^{-6}}{\omega_{j} T_{\mathrm{e}}^{1 / 2}} \Upsilon \quad \mathrm{cm}^{3} \mathrm{~s}^{-1}$,

where $\omega_{i}$ and $\omega_{j}$ are the statistical weights of the initial $(i)$ and final $(j)$ states, respectively, and $E_{i j}$ is the transition energy. The contribution of resonances may enhance the values of $\Upsilon$ over those of the background values of collision strengths $\left(\Omega_{\mathrm{B}}\right)$, especially for the forbidden transitions, by up to a factor of ten (or even more) depending on the transition and/or the temperature. Similarly, values of $\Omega$ need to be calculated over a wide energy range (above thresholds) in order to obtain convergence of the integral in Eq. (7), as demonstrated in Fig. 7 of Aggarwal \& Keenan (2008).
The temperature of maximum abundance in ionisation equilibrium for O VII is $10^{5.9} \mathrm{~K}$ (Bryans et al. 2008), while our range of energy (up to $80 \mathrm{Ryd}$ ) is sufficient to calculate values of $\Upsilon$ up to $T_{\mathrm{e}}=10^{6} \mathrm{~K}$. However, we have extended our energy range up to 140 Ryd by performing another calculation from DARC, but with a smaller $R$-matrix radius of 12.0 au. Values of $\Omega$ obtained from this calculation differ insignificantly in the $60 \leq E \leq 80$ Ryd energy range from those already described. This exercise enables us to extend the temperature range of our calculations for $\Upsilon$ up to $T_{\mathrm{e}}=2 \times 10^{6} \mathrm{~K}$.

To delineate resonances, we have performed our calculations of $\Omega$ at over 4200 energies in the threshold region. Close to thresholds ( $\sim 0.1$ Ryd above a threshold) the energy mesh is $0.001 \mathrm{Ryd}$, and away from thresholds is $0.002 \mathrm{Ryd}$. Thus care has been taken to include as many resonances as possible, and with as fine a resolution as is computationally feasible. The density and importance of resonances can be appreciated from Figs. $6 \mathrm{a}-\mathrm{f}$ and $7 \mathrm{a}-\mathrm{f}$, where we show our values of $\Omega$ in the thresholds region for some resonance transitions, namely $1-2$, $4,5,7,8,10,11,13,14,15,16$, and 17. These transitions have been chosen because Delahaye \& Pradhan (2002) have also shown similar resonances, and hence comparisons can be made between the two calculations. The density and magnitude of resonances between our calculations and those of Delahaye \& Pradhan are comparable for some transitions, such as: 1-7, 10,11 , and 17 , i.e. Figs. $6 \mathrm{~d}, \mathrm{f}$, and $7 \mathrm{a}, \mathrm{f}$, respectively. However, for some transitions, such as: 1-2, 4, 5, and 8, i.e. Figs. 6a-c, and e, respectively, resonances in our calculations are denser, particularly at energies just above the thresholds. This is mainly because fine-structure is explicitly included in the definition of channel coupling, which takes account of the relativistic effects in a more accurate way, and is particularly beneficial for splitting the terms of a state. However, the size of the Hamiltonian increases correspondingly, hence making the calculations computationally more expensive. These near threshold resonances affect the values of $\Upsilon$ particularly towards the lower end of the temperature range, which we discuss below.

Our calculated values of $\Upsilon$ are listed in Table 6 over a wide temperature range of $1.0 \times 10^{4} \leq T_{\mathrm{e}} \leq 2.0 \times 10^{6} \mathrm{~K}$, suitable for applications in solar and other plasmas. The most recent and sophisticated calculations available for comparison are by Delahaye \& Pradhan (2002), as stated in Sect. 1. They employed the $R$ - matrix code but in a Breit-Pauli approximation, which should be sufficient to account for the relativistic effects for light ions, such as $\mathrm{O}$ VII. They also resolved resonances in the thresholds region to account for their contribution in the determination of $\Upsilon$ values, and included a wide energy range for calculating values of $\Omega$ in order to ensure the convergence of the integral in Eq. (7) at all temperatures. Finally, they included contributions of all partial waves with $J \leq 17.5$ to obtain converged values of collision strengths. This limited range of partial waves is insufficient for the convergence of $\Omega$ values, particularly for transitions among the excited levels, as discussed in Sect. 5 and demonstrated in Figs. 1-3. However, this range of partial waves is fully sufficient for the convergence of $\Omega$ for all resonance transitions. Hence the $\Upsilon$ values of Delahaye \& Pradhan should be comparatively more reliable for the resonance transitions.

Delahaye \& Pradhan (2002) have not reported results for $\Omega$ or $\Upsilon$, but their results for $\Upsilon$ are available electronically on the TIPbase website (http: //vizier.u-strasbg.fr/tipbase/ home.html), as noted in Sect. 1. However, before we discuss comparisons between their and our results, we point out that the values of $\Upsilon$ shown in their Figs. 6-8 and those available on the TIPbase website are not compatible for some transitions, such as 


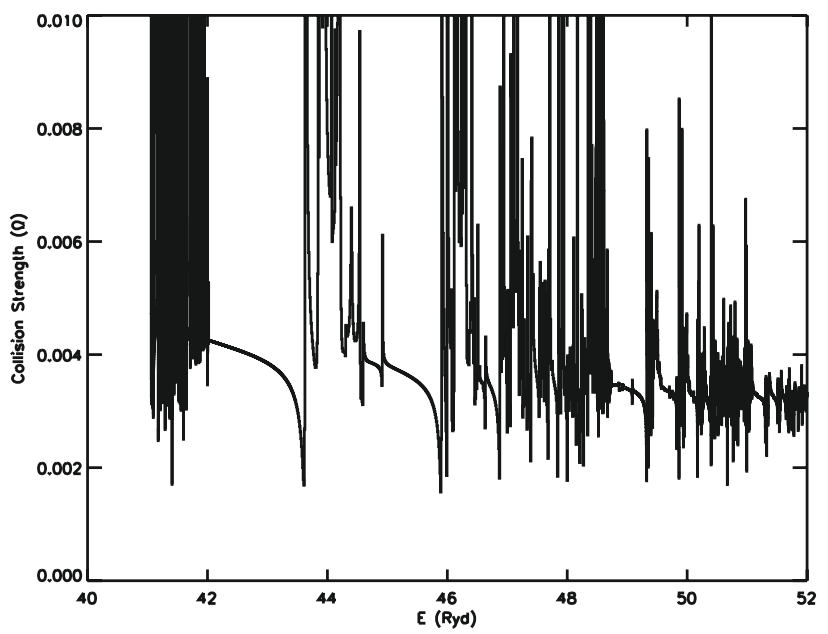

a)

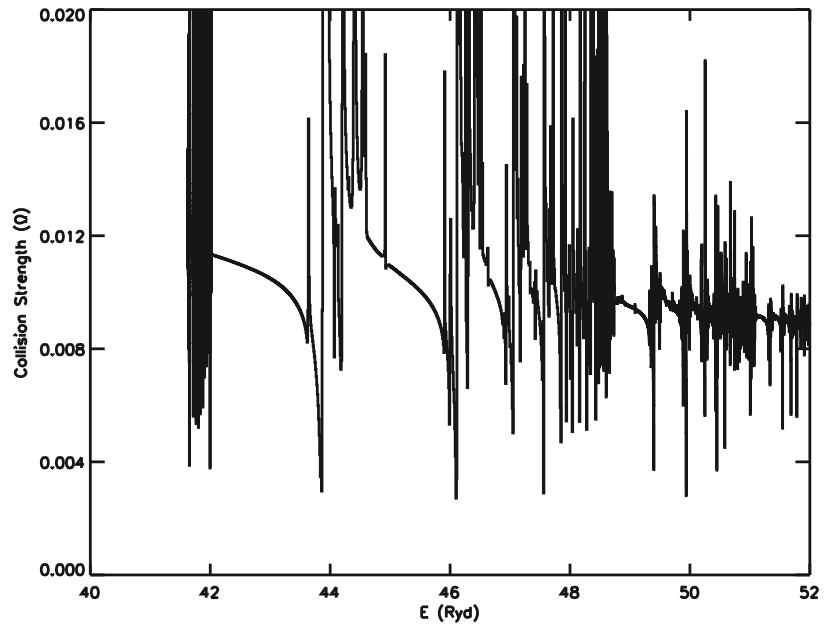

c)

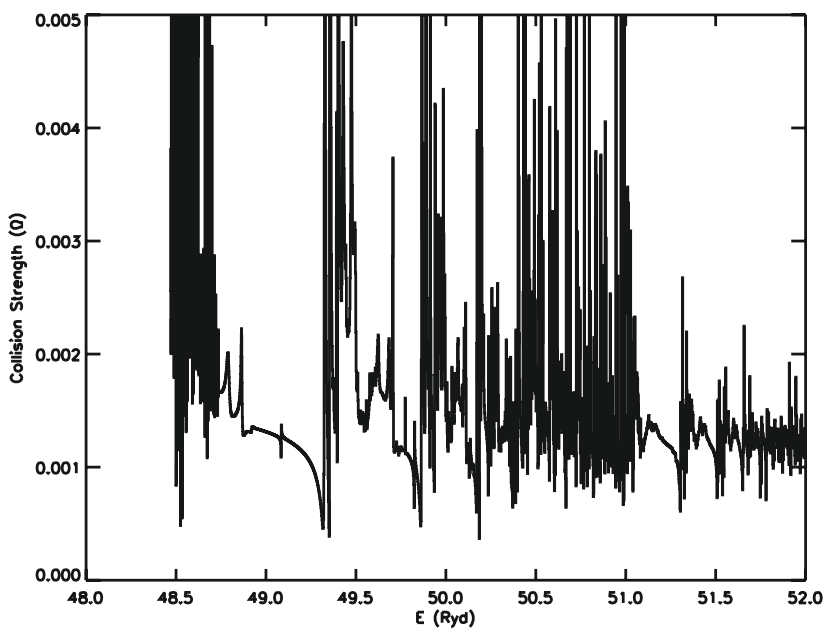

e)

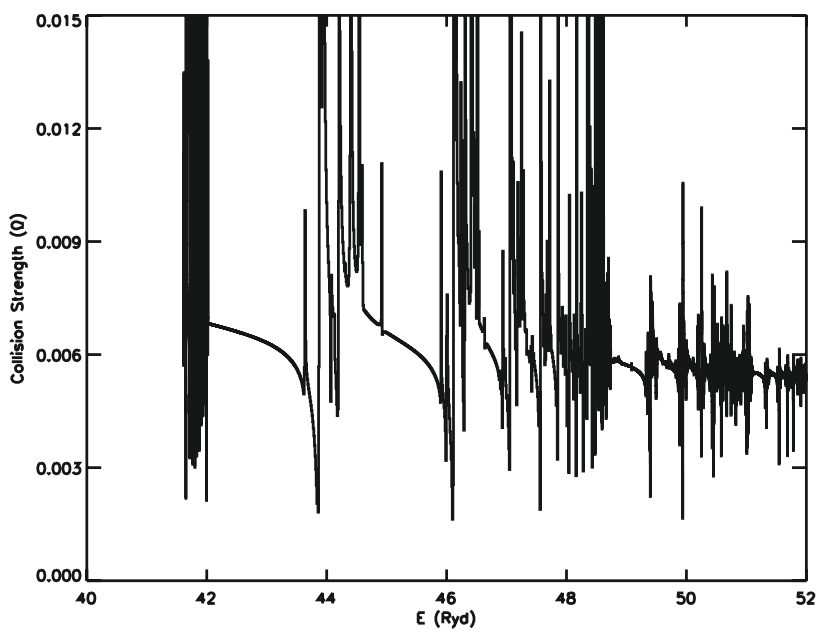

b)

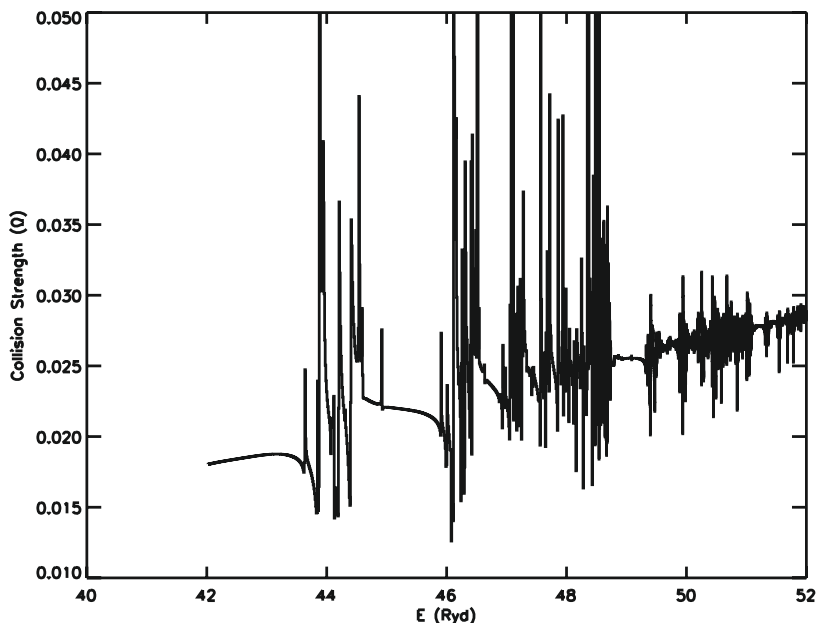

d)

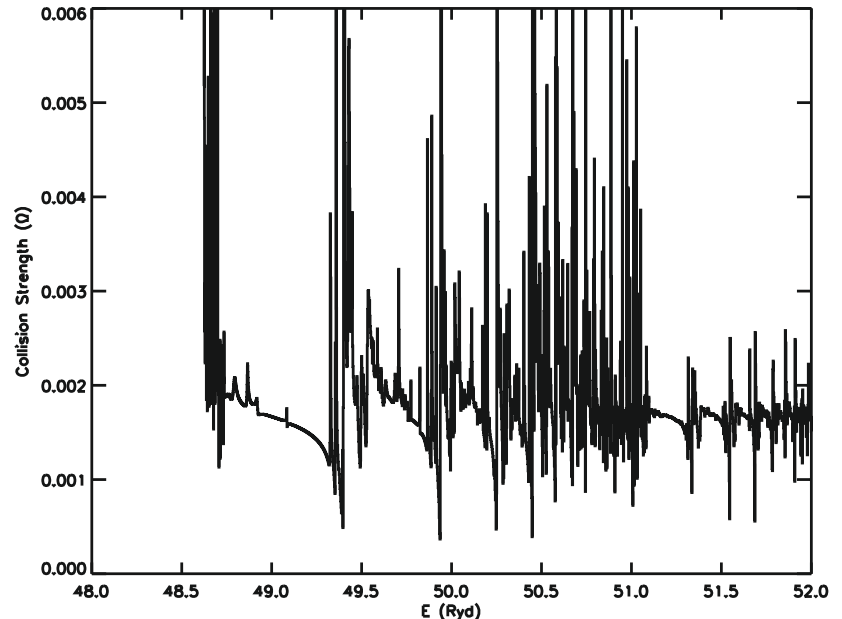

f)

Fig. 6. a) Collision strengths for the $1 \mathrm{~s}^{2}{ }^{1} \mathrm{~S}_{0}-1 \mathrm{~s} 2 \mathrm{~s}{ }^{3} \mathrm{~S}_{1}(1-2)$ transition of $\mathrm{O}$ VII. b) Collision strengths for the $1 \mathrm{~s}^{2}{ }^{1} \mathrm{~S}_{0}-1 \mathrm{~s} 2 \mathrm{p}^{3} \mathrm{P}_{1}^{\circ}(1-4)$ transition of $\mathrm{O}$ VII. c) Collision strengths for the $1 \mathrm{~s}^{2}{ }^{1} \mathrm{~S}_{0}-1 \mathrm{~s} 2 \mathrm{p}^{3} \mathrm{P}_{2}^{\circ}(1-5)$ transition of $\mathrm{O}$ VII. d) Collision strengths for the $1 \mathrm{~s}^{2}{ }^{1} \mathrm{~S}_{0}-1 \mathrm{~s} 2 \mathrm{p}{ }^{1} \mathrm{P}_{1}^{\circ}(1-7)$ transition of $\mathrm{O}$ vII. e) Collision strengths for the $1 \mathrm{~s}^{2}{ }^{1} \mathrm{~S}_{0}-1 \mathrm{~s} 3 \mathrm{~s}^{3} \mathrm{~S}_{1}(1-8)$ transition of $\mathrm{O}$ VII. f) Collision strengths for the $1 \mathrm{~s}^{2}{ }^{1} \mathrm{~S}_{0}-1 \mathrm{~s} 3 \mathrm{p}{ }^{3} \mathrm{P}_{1}^{\circ}(1-10)$ transition of $\mathrm{O}$ VII. 


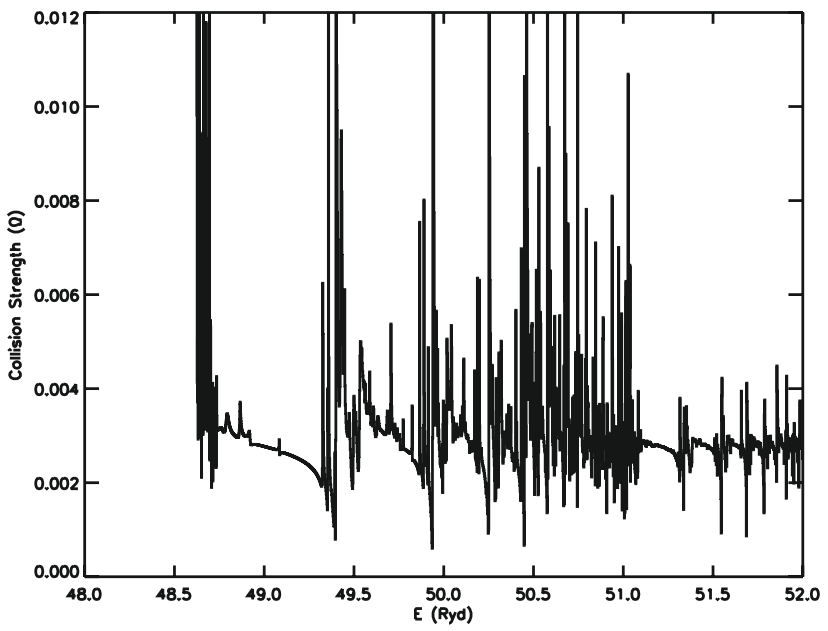

a)

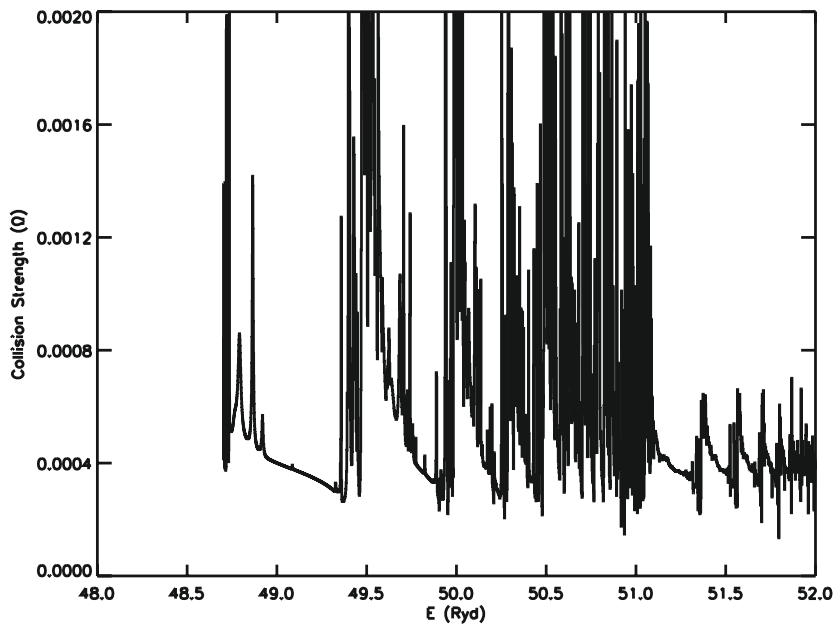

c)

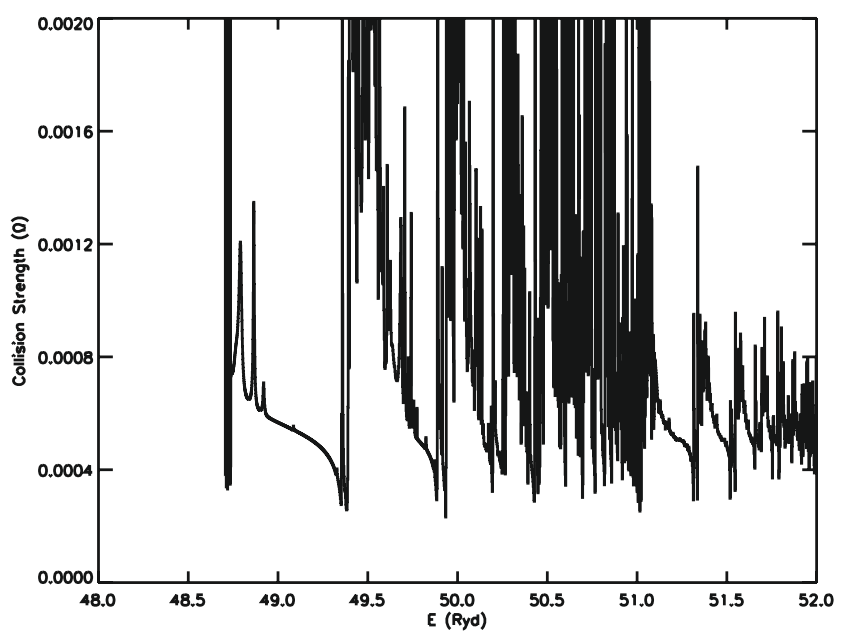

e)

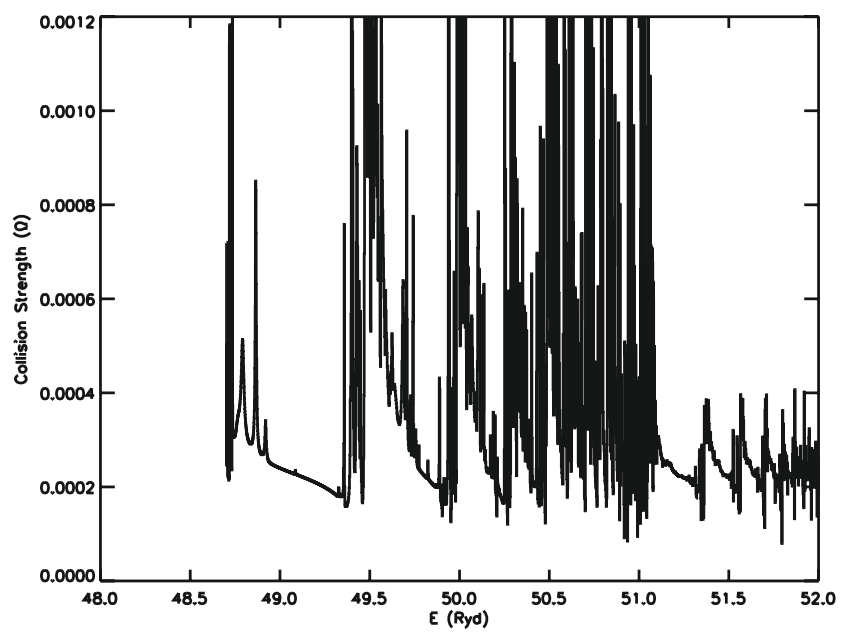

b)

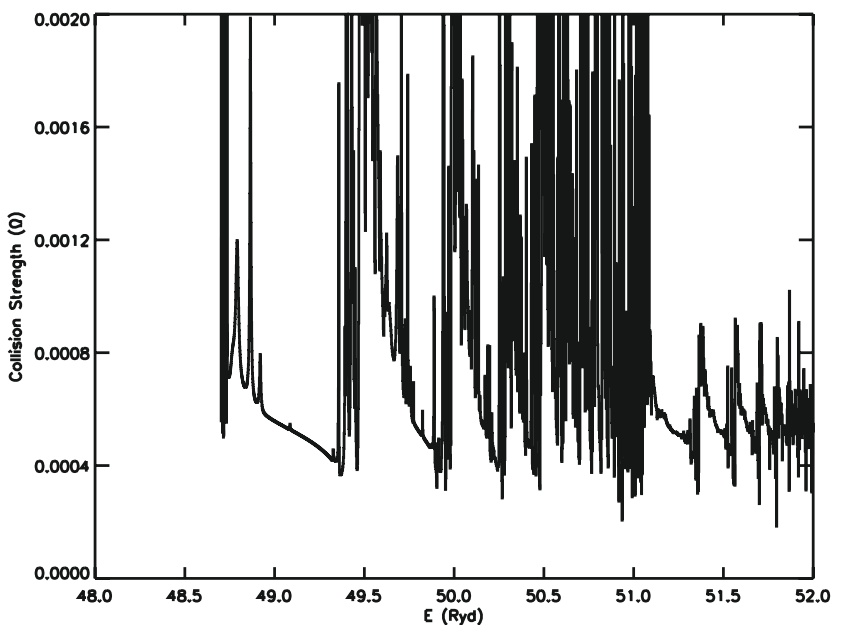

d)

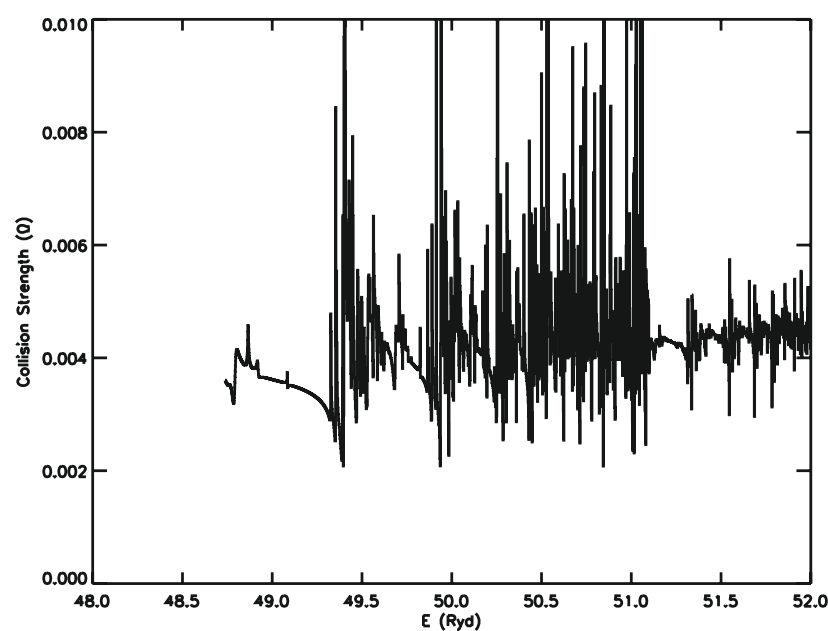

f)

Fig. 7. a) Collision strengths for the $1 \mathrm{~s}^{2}{ }^{1} \mathrm{~S}_{0}-1 \mathrm{~s} 3 \mathrm{p}^{3} \mathrm{P}_{2}^{\circ}(1-11)$ transition of $\mathrm{O}$ VII. b) Collision strengths for the $1 \mathrm{~s}^{2}{ }^{1} \mathrm{~S}_{0}-1 \mathrm{~s} 3 \mathrm{~d}^{3} \mathrm{D}_{1}(1-13)$ transition of O VII. c) Collision strengths for the $1 \mathrm{~s}^{2}{ }^{1} \mathrm{~S}_{0}-1 \mathrm{~s} 3 \mathrm{~d}^{3} \mathrm{D}_{2}(1-14)$ transition of $\mathrm{O}$ VII. d) Collision strengths for the $1 \mathrm{~s}^{2}{ }^{1} \mathrm{~S}_{0}-1 \mathrm{~s} 3 \mathrm{~d}^{3} \mathrm{D}_{3}(1-15)$ transition of $\mathrm{O}$ VII. e) Collision strengths for the $1 \mathrm{~s}^{2}{ }^{1} \mathrm{~S}_{0}-1 \mathrm{~s} 3 \mathrm{~d}{ }^{1} \mathrm{D}_{2}(1-16)$ transition of $\mathrm{O}$ VII. f) Collision strengths for the $1 \mathrm{~s}^{2}{ }^{1} \mathrm{~S}_{0}-1 \mathrm{~s} 3 \mathrm{p}^{1} \mathrm{P}_{1}^{\circ}(1-17)$ transition of $\mathrm{O}$ VII. 


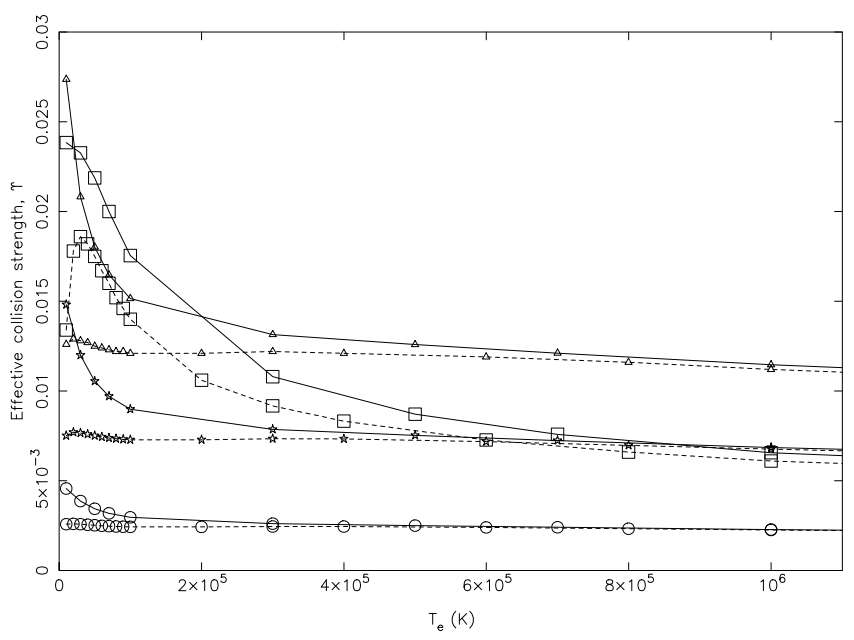

Fig. 8. Comparison of effective collision strengths from our calculations from DARC (continuous curves) and those of Delahaye \& Pradhan (2002: broken curves) for the 1-2 (squares: $1 \mathrm{~s}^{2}{ }^{1} \mathrm{~S}_{0}-1 \mathrm{~s} 2 \mathrm{~s}^{3} \mathrm{~S}_{1}$ ), 1-3 (circles: $1 \mathrm{~s}^{2}{ }^{1} \mathrm{~S}_{0}-1 \mathrm{~s} 2 \mathrm{p}{ }^{3} \mathrm{P}_{0}^{\circ}$ ), $1-4$ (stars: $1 \mathrm{~s}^{2}{ }^{1} \mathrm{~S}_{0}-1 \mathrm{~s} 2 \mathrm{p}^{3} \mathrm{P}_{1}^{\circ}$ ), and $1-5$ (triangles: $1 \mathrm{~s}^{2}{ }^{1} \mathrm{~S}_{0}-1 \mathrm{~s} 2 \mathrm{p}^{3} \mathrm{P}_{2}^{\circ}$ ) transitions of $\mathrm{O}$ VII.

4-6 (1s $\left.2 \mathrm{p}^{3} \mathrm{P}_{1}^{\circ}-1 \mathrm{~s} 2 \mathrm{~s}{ }^{1} \mathrm{~S}_{0}\right), 4-13\left(1 \mathrm{~s} 2 \mathrm{p}{ }^{3} \mathrm{P}_{1}^{\circ}-1 \mathrm{~s} 3 \mathrm{~d}{ }^{3} \mathrm{D}_{1}\right)$, and $8-14$ $\left(1 \mathrm{~s} 3 \mathrm{~s}^{3} \mathrm{~S}_{1}-1 \mathrm{~s} 3 \mathrm{~d}^{3} \mathrm{D}_{2}\right)$. For the $4-13$ transition we assume this corresponds to $1 \mathrm{~s} 2 \mathrm{p}{ }^{3} \mathrm{P}_{1}^{\circ}-1 \mathrm{~s} 3 \mathrm{~d}{ }^{3} \mathrm{D}_{1}$, rather than $1 \mathrm{~s} 2 \mathrm{p}{ }^{3} \mathrm{P}_{1}^{\circ}-2{ }^{3} \mathrm{D}_{1}$ as labelled by Delahaye \& Pradhan. Nevertheless, a comparison of their results with ours indicates that the $\Upsilon$ values listed on the website are correct, and therefore we will discuss comparisons with those results alone.

A comparison between our $\Upsilon$ values and those of Delahaye \& Pradhan (2002) shows differences of over $20 \%$ for almost all ( $89 \%$ to be precise) the 465 common transitions among the lowest 31 levels, in the common temperature range of $1.0 \times 10^{4} \leq$ $T_{\mathrm{e}} \leq 2.0 \times 10^{6} \mathrm{~K}$. Since resonance transitions are the most important and probably the most accurate among those calculated by Delahaye \& Pradhan, as discussed above, we focus on a comparison for these transitions. Temperatures towards the lower end are particularly sensitive to the presence (or absence) of those resonances which are near the thresholds, as shown in Fig. 6 (a-c and e-f). To demonstrate this, we compare the two sets of $\Upsilon$ for the 1-2, 3, 4, and 5 transitions in Fig. 8. For all these (and many other) transitions the discrepancy is the largest (up to a factor of two) at the lowest temperature, and decreases with increasing temperature. Our larger values of $\Upsilon$ for such transitions are understandable for the reasons discussed above, and are clearly due to the denser resonances we observe in our calculations. However, transitions involving levels 23 and higher show even larger discrepancies of up to an order of magnitude, and particularly notable are four, namely 1-24, 27, 29, and 31 . Since the discrepancy is the largest for the $1-24\left(1 \mathrm{~s}^{2}{ }^{1} \mathrm{~S}_{0}-1 \mathrm{~s} 4 \mathrm{~d}\right.$ $\left.{ }^{3} \mathrm{D}_{2}\right)$ and $1-27\left(1 \mathrm{~s}^{2}{ }^{1} \mathrm{~S}_{0}-1 \mathrm{~s} 4 \mathrm{f}^{3} \mathrm{~F}_{3}^{\circ}\right)$ transitions, we focus our comparison on these to understand the differences.

In Fig. 9 we show our values of $\Upsilon$ and those of Delahaye \& Pradhan (2002) for both the 1-24 and 1-27 transitions. Since our values of $\Upsilon$ are consistently higher over the entire temperature range, the first suspicion is that the differences are due to resonances, because we have included an additional 18 levels of the $n=5$ configurations, whereas Delahaye \& Pradhan have not. These transitions do show resonances over the entire threshold energy range, as shown for illustration in Fig. 10 for the 124 transition for which the discrepancy is the largest. However, these resonances are neither dense nor very large in magnitude,

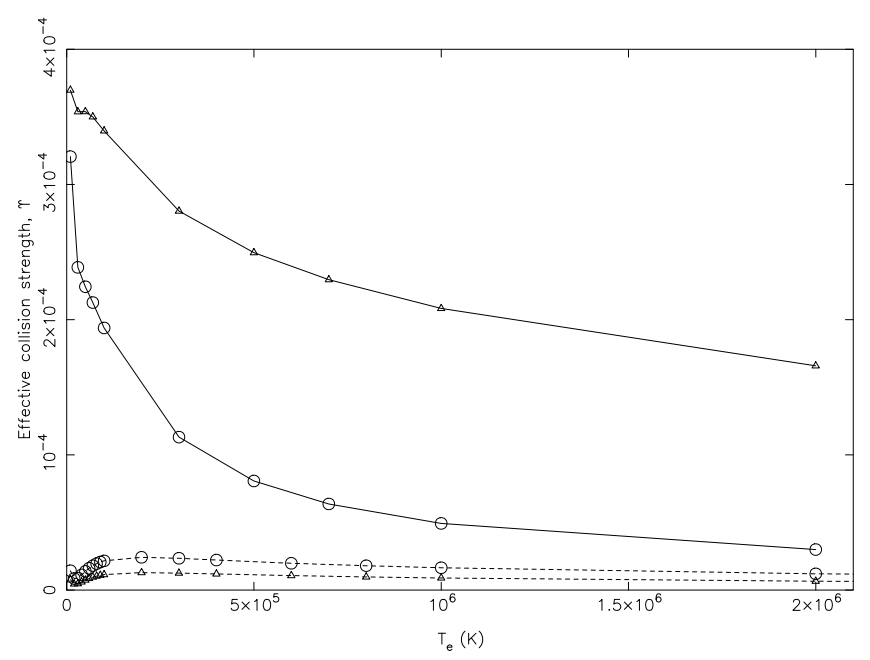

Fig. 9. Comparison of effective collision strengths from our calculations from DARC (continuous curves) and those of Delahaye \& Pradhan (2002: broken curves) for the 1-24 (triangles: $1 \mathrm{~s}^{2}{ }^{1} \mathrm{~S}_{0}-1 \mathrm{~s} 4 \mathrm{~d}^{3} \mathrm{D}_{2}$ ) and 1-27 (circles: $1 \mathrm{~s}^{2}{ }^{1} \mathrm{~S}_{0}-1 \mathrm{~s} 4 \mathrm{f}^{3} \mathrm{~F}_{3}^{\circ}$ ) transitions of $\mathrm{O}$ VII.

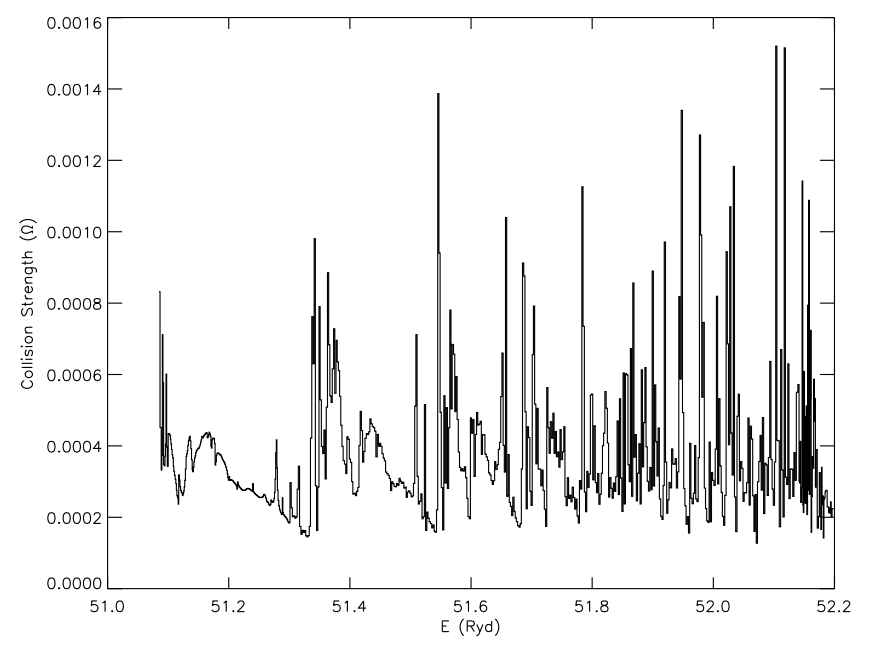

Fig. 10. Collision strengths for the $1 \mathrm{~s}^{2}{ }^{1} \mathrm{~S}_{0}-1 \mathrm{~s} 4 \mathrm{~d}^{3} \mathrm{D}_{2}(1-24)$ transition of O VII.

and therefore the effect on the determination of $\Upsilon$ values is not very significant, especially at temperatures above $10^{5} \mathrm{~K}$. Hence, the differences between the two sets of $\Upsilon$ values probably arise from the differences in the corresponding values of $\Omega$. Unfortunately, Delahaye \& Pradhan have not reported their results for $\Omega$, except for a few transitions in graphical form. However, for a majority of the transitions, and particularly the resonance lines, there is no (large) discrepancy between our calculations from DARC and FAC, as discussed already in Sect. 5 and may also be noted from Table 5. Furthermore, our values of $\Omega$ and $\Upsilon$ decrease with increasing energy (temperature), as both of these are forbidden transitions. However, this is not so much apparent in the calculations of Delahaye \& Pradhan. Therefore, we have confidence in our results. Finally, we note that even over the higher temperature range $\left(1.0 \times 10^{5} \leq T_{\mathrm{e}} \leq 2.0 \times 10^{6} \mathrm{~K}\right)$, differences between our values of $\Upsilon$ and those of Delahaye $\&$ Pradhan are over $20 \%$ for about $80 \%$ of the transitions in common. Differences of about an order of magnitude are common for many transitions, but are particularly large (up to two orders of magnitude) for six, namely 2-24, 25; 21-24, 23-26, 24-27, and 27-30. Most of the differences are (perhaps) due to 
the differences in the corresponding values of $\Omega$, because the limited range of partial waves $(J \leq 17.5)$ adopted by Delahaye $\&$ Pradhan is inadequate for the convergence of $\Omega$ for a large number of forbidden and most of the allowed transitions among excited levels, as discussed earlier in Sect. 5.

\section{Conclusions}

In this paper we have presented results for energy levels and radiative rates for four types of transitions (E1, E2, M1, and M2) among the lowest 49 levels of O VII belonging to the $n \leq 5$ configurations. Additionally, lifetimes of all the levels have been reported, although measurements are available for only a few, for which there is no discrepancy between theory and experiments. Based on a variety of comparisons, our energy levels are assessed to be accurate to better than $0.5 \%$, and the results for radiative rates, oscillator strengths, line strengths, and lifetimes are assessed to be accurate to better than $20 \%$ for a majority of the strong transitions (levels). Similarly, the accuracy of our results for collision strengths and effective collision strengths is estimated to be better than $20 \%$ for a majority of the transitions. This accuracy estimate is based on a comparison between two independent calculations performed with the DARC and FAC codes. Additionally, we have considered a large range of partial waves in order to achieve the convergence of values of $\Omega$ at all energies, included a wide energy range in order to accurately calculate the values of $\Upsilon$ up to $T_{\mathrm{e}}=2.0 \times 10^{6} \mathrm{~K}$, and resolved resonances in a fine energy mesh in order to account for their contributions. Hence, overall improvements have been made over the earlier available $\Upsilon$ results of Delahaye \& Pradhan (2002), which differ from the present calculations by over an order of magnitude for many transitions. Finally, we have also included the additional 18 levels of the $n=5$ configurations, which have helped to improve the accuracy of the values of $\Upsilon$ for all those transitions whose levels belong to the $n \leq 4$ configurations. Similarly, our present results for transitions involving the levels of the $n=5$ configurations can be further improved by the inclusion of the levels of the $n=6$ configurations. We believe the present set of complete results for radiative and excitation rates are the most reliable currently available, and will be highly useful for the modelling of a variety of plasmas.

Acknowledgements. This work has been financed by the Engineering and Physical Sciences and Science and Technology Facilities Councils of the United Kingdom, and F.P.K. is grateful to A. W. E. Aldermaston for the award of a William Penney Fellowship. We thank Dr. P. H. Norrington for providing his revised GRASP and DARC codes prior to publication.

\section{References}

Acton, L. W., Catura, R. C., Meyerott, A. J., Wolfson, C. J., \& Culhane, J. L. 1972, Sol. Phys., 26, 183

Aggarwal, K. M., \& Keenan, F. P. 2008, Eur. Phys. J., D 46, 205

Aggarwal, K. M., Tayal, V., Gupta, G. P., \& Keenan, F. P. 2007, ADNDT, 93, 615

Aggarwal, K. M., Hamada, K., Igarashi, A., et al. 2008, A\&A, 484, 879

Baker, S. C. 1973, J. Phys. B, 6, 709

Berrington, K. A., Eissner, W. B., \& Norrington, P. H. 1995, Comput. Phys. Commun., 92, 290

Bryans, P., Landi, E., \& Savin, D. W. 2008, ApJ, in press

[arXiv: 0805.3302]

Delahaye, F. \& Pradhan, A. K. 2002, J. Phys. B, 35, 3377

Dere, K. P., Landi, E., Young, P. R., \& Del Zanna G. 2001, ApJS, 134, 331

Eissner, W., Jones, M., \& Nussbaumer, H. 1974, Comput. Phys. Commun., 8, 270

Gabriel, A. H., \& Jordan, C. 1969, MNRAS, 145, 241

Grant, I. P., McKenzie, B. J., Norrington, P. H., Mayers, D. F., \& Pyper, N. C. 1980, Comput. Phys. Commun., 21, 207

Gu, M. F. 2003, ApJ, 582, 1241

Hibbert, A. 1996, Phys. Scr., T65, 104

Hibbert, A. 2000, AIP Conf. Proc., 543, 242

Isler, R. C., Jupen, C., \& Martinson, I. 1993, Phys. Scr., 47, 32

Keenan, F. P., Kingston, A. E., \& McKenzie, D. L. 1985, ApJ, 291, 855

McKenzie, D. L., Landecker, P. B., Broussard, R. M., et al. 1980, ApJ, 241, 409

Phillips, K. J. H., Mewe, R., Harra-Murnion, L. K., et al. 1999, A\&AS, 138, 381

Savukov, I. M., Johnson, W. R., \& Safranova, U. I. 2003, ADNDT, 85, 83

Träbert, E., Heckmann, P. H., \& Buttlar, H. V. 1977, Z. Phys. A, 280, 11

Winkler, P. F., Clark, G. W., Markert, T. H., Petre, R., \& Canizares, C. R. 1981, ApJ, 245, 574 